\title{
Temperature- and doping-dependent optical absorption in the small-polaron system $\operatorname{Pr}_{1-x} \mathrm{Ca}_{x} \mathrm{MnO}_{3}$
}

\author{
S. Mildner, ${ }^{1, *}$ J. Hoffmann, ${ }^{1}$ P. E. Blöchl, ${ }^{1,2}$ S. Techert, ${ }^{3,4,5}$ and C. Jooss ${ }^{1}$ \\ ${ }^{1}$ Institute for Material Physics, University of Göttingen, Friedrich-Hund-Platz 1, D-37077 Göttingen, Germany \\ ${ }^{2}$ Institute for Theoretical Physics, University of Clausthal, Leibnizstrasse 10, D-38678 Clausthal-Zellerfeld, Germany \\ ${ }^{3}$ Max-Planck-Institute for Biophysical Chemistry, Am Faßberg 1, D-37077 Göttingen, Germany \\ ${ }^{4}$ FS-SCS Deutsches Elektronensynchrotron DESY, Notkestrasse 85, D-22607 Hamburg, Germany \\ ${ }^{5}$ Institute for X-ray Physics, University of Goettingen, Friedrich-Hund-Platz 1, D-37077 Göttingen, Germany
}

(Received 1 March 2015; revised manuscript received 18 May 2015; published 24 July 2015)

\begin{abstract}
Small polaron optical properties are studied comprehensively in thin film samples of the narrow bandwidth manganite $\operatorname{Pr}_{1-x} \mathrm{Ca}_{x} \mathrm{MnO}_{3}$ by optical absorption spectroscopy as a function of doping and temperature. A broad near infrared double-peak absorption band in the optical conductivity spectras is observed and interpreted in the framework of photon-assisted small polaron intersite hopping and on-site Jahn-Teller excitation. Application of quasiclassical small polaron theory to both transitions allows an approximate determination of polaron specific parameters like the polaron binding energy, the characteristic phonon energy, as well as the Jahn-Teller splitting energy as a function of temperature and doping. Based on electronic structure calculations, we consider the impact of the hybridization of $\mathrm{O} 2 p$ and $\mathrm{Mn} 3 d$ electronic states on the Jahn-Teller splitting and the polaron properties. The interplay between hopping and Jahn-Teller excitations is discussed in the alternative pictures of mixed valence $\mathrm{Mn}^{3+} / \mathrm{Mn}^{4+}$ sites (Jahn-Teller polaron) and equivalent $\mathrm{Mn}^{(3+x)+}$ sites (Zener polaron). We give a careful evaluation of the estimated polaron parameters and discuss the limitations of small polaron quasiclassical theory for application to narrow bandwidth manganites.
\end{abstract}

DOI: 10.1103/PhysRevB.92.035145

PACS number(s): 71.38.-k, 75.47.Lx, 78.20.Ci, 71.27.+a

\section{INTRODUCTION}

Polarons, i.e., quasiparticles composed by electronic charge carriers and phonons, are a key transport feature in doped manganites. Many properties of manganites such as hoppinglike electric transport behavior [1,2], charge ordering [3,4], as well as colossal resistance effects [5-7] involve polaronic effects [8,9]. A polaron forms as a consequence of a significant charge-lattice interaction, i.e., the electric charge carrier distorts its surrounding lattice resulting in the formation of a potential well. In the case of a small polaron this potential well localizes the charge carrier at a single lattice site [10]. A large polaron forms if the lattice distortion spreads over several lattice sites [11-13].

Optical absorption spectroscopy in the near-infrared (NIR) region allows studying the properties of polaron excitations [14-17]. For example, Quijada et al. investigated the temperature-dependent optical properties of $\mathrm{La}_{2 / 3} \mathrm{Ca}_{1 / 3} \mathrm{MnO}_{3}$, $\mathrm{La}_{2 / 3} \mathrm{Sr}_{1 / 3} \mathrm{MnO}_{3}$, and $\mathrm{Nd}_{2 / 3} \mathrm{Sr}_{1 / 3} \mathrm{MnO}_{3}$ thin films. These compounds undergo a temperature driven metal-insulator phase transition which is accompanied by a huge spectral weight transfer in the NIR range [16]. The authors observed that phases with small polaron hopping conductivity involve an absorption maximum at $\hbar \omega_{\max } \approx 1-2 \mathrm{eV}$. Phases with metal-like conductivity reveal a Drude-like feature in the infrared range, which is interpreted in the framework of large polaron transport. Similar trends in the crossover from small to large polaron optical absorption are observed by Hartinger et al. [14].

Okimoto et al. presented temperature-dependent optical conductivity spectra of low bandwidth $\operatorname{Pr}_{1-x} \mathrm{Ca}_{x} \mathrm{MnO}_{3}(x=$ $0.4)$ single crystals up to photon energies of $\hbar \omega \leqslant 1.2 \mathrm{eV}$

\footnotetext{
*sraabe@ump.gwdg.de
}

obtained from NIR reflectivity data [18]. Their data indicate a broad absorption feature around $\hbar \omega \approx 1 \mathrm{eV}$ which was interpreted in terms of intersite $d$ - $d$ transfer transitions of the charge carriers. Evidence for small polaron absorption in $\operatorname{Pr}_{1-x} \mathrm{Ca}_{x} \mathrm{MnO}_{3}(x=0.33)$ is presented by Saucke et al. in $p n$ manganite heterojunctions [19].

Commonly, the NIR optical conductivity of manganites are interpreted in the framework of transition between $\mathrm{Mn}$ $3 d$ states where $\mathrm{O} 2 p$ states near the Fermi level are not considered [18,20]. However, recent literature proves that O $2 p$ states are involved in NIR optical excitation [21]. Moreover, studies of the electronic structure of manganites reveal a strong contribution of $\mathrm{O} 2 p$ states to the upper valence band edge [3,22-25].

Among the numerous studies on the optical properties of manganites, systematic studies of the doping dependence are rare [26]. Moreover, despite the recognition that the NIR absorption in manganites carries polaron signature $[14,16]$, analytical expressions for the optical conductivity of polarons provided by polaron theory, for instance in Refs. [27,28], are rarely applied to experimental data. To our knowledge, the only works in which polaron theory was used to fit optical spectra of manganites and extract polaron properties are Refs. [14,15,29,30]. However, a systematic study of the temperature and doping dependence of polaron properties is not provided in these works.

In order to systematically study the temperature and dopingdependent optical properties of small polarons, which are formed out of a $\mathrm{O} 2 p$-Mn $3 d\left(e_{g}\right)$ hybrid band, we present temperature-dependent optical conductivity spectra as well as dc conductivity data of $\operatorname{Pr}_{1-x} \mathrm{Ca}_{x} \mathrm{MnO}_{3}$ (PCMO) thin films in the doping range of $0 \leqslant x \leqslant 0.8$. The polaronic nature of the double peak excitation in the NIR is analyzed by quasiclassical small polaron theory. This allows 
for the approximate determination of the temperature and doping-dependent polaron binding energy and the involved phonon energies. The observed double peak structure is discussed and interpreted in the framework of intersite polaron hopping transitions and on-site Jahn-Teller (JT) like excitations. Electronic structure calculations are presented which allow for discussion of electronic band structure effects on polaron excitation, in particular the effect of hybridization of oxygen $2 p$ and manganese $3 d$ states at the upper valence band edge. We critically discuss the applicability of quasiclassical small polaron theory and derive conclusions on the coupled electron-lattice dynamics in manganites which may initiate further time-resolved experimental studies.

\section{EXPERIMENT}

Epitaxial PCMO thin films $(x=0-0.8)$ were prepared by reactive ion-beam sputtering on single crystalline (001)oriented $\mathrm{MgO}$ substrate. Sputter targets of the selected compositions $(x=0,0.2,0.35,0.5,0.8)$ were fabricated by a solid state reaction from stoichiometric mixtures of the dry $\mathrm{Pr}_{6} \mathrm{O}_{11}, \mathrm{CaCO}_{3}$, and $\mathrm{Mn}_{2} \mathrm{O}_{3}$ powders. Repeated milling and calcinating lead to pure perovskite phases as revealed by $\mathrm{x}$-ray diffractometry. Finally, PCMO targets are cold-pressed and sintered in air at $1370 \mathrm{~K}$ for $48 \mathrm{~h}$. The ion-beam sputtering process is performed in a gas mixture of xenon as working gas and oxygen as reactive gas with partial pressures of $10^{-4} \mathrm{mbar}$ at a deposition temperature of $750{ }^{\circ} \mathrm{C}$. The thin film samples with the doping levels $x=0.2,0.35$, and 0.5 have been postannealed in air at temperatures of $800{ }^{\circ} \mathrm{C}(x=0.35,0.5)$ for $10 \mathrm{~h}$ and $1000^{\circ} \mathrm{C}(x=0.2)$ for $1-2 \mathrm{~h}$. The thin films have a thicknesses of $t=100-150 \mathrm{~nm}$. All samples with $x<0.8$ reveal epitaxial growth with preferential (001) orientation as well as (110) orientation due to twinning. In contrast, the sample with $x=0.8$ shows (112)-oriented growth on $\mathrm{MgO}$ under the same deposition conditions.

Temperature-dependent two-point resistance measurements are performed with a source voltage of $1 \mathrm{~V}$ using a closed cycle compressed helium cryostat. For colossal magnetoresistance (CMR) measurements a Physical Properties Measurement System (PPMS) from Quantum Design is used. Field cooled resistance $R(T)$ is measured in an applied magnetic field of $B=9 \mathrm{~T}$. The subsequent $R(T)$ heating curve is measured in zero field.

Temperature-dependent optical absorption measurements were performed in a Cary Varian 5e spectrometer with unpolarized light in a wavelength range of $250-3300 \mathrm{~nm}$ at temperatures between $T=80$ and $300 \mathrm{~K}$. To provide temperature control the thin film samples were installed in an Oxford Fast track Microstat $\mathrm{H} 2$ gas flow cryogenic system cooled with liquid nitrogen. The absorption of the $\mathrm{MgO}$ substrate was subtracted via reference measurements of a blank substrate. The real part of the optical conductivity is determined from the measured absorption coefficient $\alpha(\omega)$ via $\sigma(\omega)=\varepsilon_{0} c_{0} \alpha(\omega)$ in the limit of optically dense thin films $t \ll c_{0} / n \omega$, where $c_{0}$ is the light velocity and $n$ is the refractive index. This approximation is particularly fulfilled in the NIR range.

We performed density-functional calculations [31,32] with the projector augmented wave method as implemented in the CP-PAW code package [http://www2.pt.tu-clausthal.de/ paw/] [33]. We used a local hybrid density functional [34-36], where a fraction of the exchange in the PBE functional is replaced by an explicit Fock term [37]. The factors are 0.1 for $\mathrm{Ca}, \mathrm{Mn}$, and $\mathrm{O}$ atoms and 0.15 for Pr. For the Mn $d$ states this amounts to a screened $U$ parameter of $2.3 \mathrm{eV}$ and a screened $J$ parameter of $0.1 \mathrm{eV}$. In the local approximation, the exchange correction is limited to the on-site terms in a local orbital basis set.

\section{QUASICLASSICAL THEORY OF SMALL POLARON HOPPING}

The polaron state is commonly described by three coupling parameters $\alpha, \lambda$, and $\gamma$ which compare the energy scales of the electron-phonon coupling energy $g$, the phonon vibrational energy $\hbar \omega_{0}$, and the electron kinetic energy $D$ [38],

$$
\alpha \equiv \frac{g}{\hbar \omega_{0}}=\sqrt{\frac{E_{P}}{\hbar \omega_{0}}}, \quad \lambda \equiv \frac{E_{P}}{D}, \quad \text { and } \quad \gamma \equiv \frac{\hbar \omega_{0}}{J} \text {. }
$$

Small polarons are formed if the electron-phonon coupling energy $g$ is larger than the energy of the involved phonon mode $\hbar \omega_{0}$ and the polaron binding energy $E_{P}$ is larger than the kinetic energy $D$ of the electrons ( $2 D$ denotes the electronic bandwidth), i.e., $\lambda \geqslant 1$.

Both conditions are fulfilled in the low bandwidth manganite PCMO in zero magnetic field [6]. The adiabicity parameter $\gamma$ indicates whether the coupled electron-lattice dynamics is governed by the electron motion (adiabatic, $\gamma \rightarrow 0$ ) or by the lattice vibration (antiadiabatic, $\gamma \rightarrow \infty$ ).

Small polaron motion is commonly discussed in the framework of the simple Holstein two-site model (Fig. 1), which describes a single electron in a molecule consisting of two equivalent lattice sites where the electron can hop between the two sites. In the strong coupling limit $\lambda>1$, the electronlattice interaction leads to a reorganization of the electronic energy levels and a lattice distortion at the occupied site. The distortion is described by the configurational coordinate $q_{i}$ which characterizes the antisymmetric relaxation of the two sites. It has the value -1 and +1 if the polaron is formed on the left or right site, respectively. In the symmetric state $\left(q_{1}=\right.$ $q_{2} \equiv 0$ ) the electronic states on the two sites are degenerate. A finite electronic overlap integral $J>0$ between the two sites lifts the degeneracy at $q_{1}=q_{2} \equiv 0$ by a splitting energy of $2 J$. For a three-dimensional crystal, the tight-binding theory relates the overlap integral $J$ and the electronic bandwidth $2 D$ via $J=D / Z$. In an octahedral environment the number of nearest neighbor sites is $Z=6$.

Within the framework of the quasiclassical Holstein model, the polaron dynamics is approximated by a combination of quantum-mechanical electron dynamics and a classical vibrational field. In the adiabatic limit, the temperature-dependent resistivity due to thermally activated small polaron hopping is [39]

$$
\rho(T)=\rho_{0} T \exp \left(\frac{E_{A}}{k_{B} T}\right) .
$$

The activation energy $E_{A}$ is related to the polaron binding energy and the transfer integral [13]

$$
E_{A}=\frac{1}{2} E_{P}-J .
$$




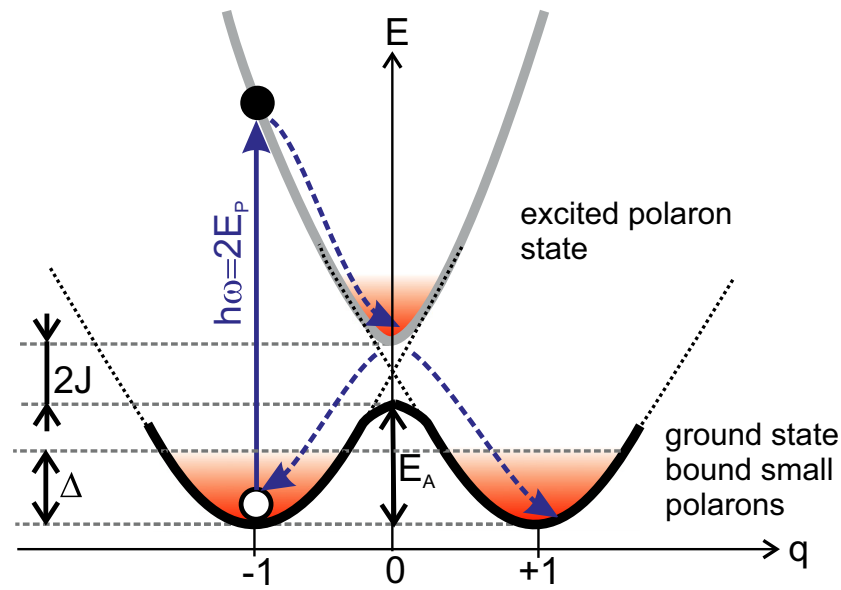

FIG. 1. (Color online) Scheme of small polaron excitations presented in the framework of the two-site model in the adiabatic limit using parabolic potential surfaces to visualize a Franck-Condon-like excitation at $\hbar \omega \approx 2 E_{P}$ and two relaxation channels, leading to either hopping transfer or on-site relaxation. At low temperatures, the broadening of the electronic levels $\Delta$ is determined by zero-point phonon fluctuations. The potential energy of such two-site system is determined by the electronic energy gain $E_{\text {el }} \propto-g \sum f_{i} q_{i}$ (where $f_{i}$ denotes the occupation of site $i, \sum f_{i}=1$ ) and the energy expended for the distortion of the lattice $E_{\text {dist }} \propto+\hbar \omega_{0} \sum q_{i}^{2}$ [40]. Hence, the potential energy at each site as a function of $q_{i}$ is described by parabolic potential curves depending on the occupation $0 \leqslant f_{i} \leqslant 1$ of the one-electron orbital. Note that $q$ denotes the relative distortion of both sites.

Figure 1 also illustrates the Franck-Condon-like (adiabatic) excitation of a bound small polaron from its ground state [40]. The ground state is broadened due to phonon fluctuations with the energy variance $\Delta$. If the electronic overlap integral is small so that $D=Z J \ll \Delta$ is fulfilled [Eq. (6)], the optical excitation of a small polaron requires twice the polaron binding energy $E_{P}$. From the excited state the polaron may relax to ground state either on-site or to a nearest neighbor site, resulting in a hopping transfer.

The quasiclassical theory on the basis of the Holstein model also provides analytical expressions for the frequencydependent intraband absorption of polarons [11,27,28]. In the adiabatic regime, the real part of the frequency-dependent optical conductivity of noninteracting small polarons is given by (Eq. 29 in Ref. [27]), see also Refs. [11,28,41],

$$
\sigma(\omega, T)=\sigma(0, T) \frac{\sinh \left(2 \hbar^{2} \omega_{\max } \omega / \Delta^{2}\right)}{2 \hbar^{2} \omega_{\max } \omega / \Delta^{2}} \exp \left[-\frac{(\hbar \omega)^{2}}{\Delta^{2}}\right],
$$

i.e., the absorption reveals a skewed Gaussian peak with a maximum at $\hbar \omega_{\max }$. The prefactor $\sigma(0, T)$ is the electrical dc conductivity arising from phonon-assisted polaron hopping motion. Equation (4) requires that the width of the absorption peak is governed by the phonon induced broadening $\Delta$ of the involved energy levels,

$$
\Delta^{2}=8 E_{P} E_{\mathrm{vib}},
$$

i.e., the effect of the electronic bandwidth $2 D$ is small $(D \ll \Delta)$. At zero-point temperature the vibrational energy
$E_{\mathrm{vib}}$ corresponds to $E_{\mathrm{vib}}=\hbar \omega_{0} / 2$. In the high temperature regime $\left(k T \gg \hbar \omega_{0}\right) E_{\mathrm{vib}}$ is equal to the thermal energy, i.e., $E_{\mathrm{vib}}=k T$.

A rough estimate for PCMO based on $\hbar \omega_{0}=40-70 \mathrm{meV}$ [42,43], and $2 D \sim 850 \mathrm{meV}$ (Sec. V A) gives $0 \ll \gamma<1$, i.e., $\gamma$ is near the adiabatic to antiadiabatic crossover and, thus, nonadiabatic effects have to be considered.

However, dynamical mean-field calculations (DMFT) for small polaron optical conductivity show that small polaron absorption gives rise to an absorption peak in the NIR with an absorption maximum at [28]

$\hbar \omega_{\max }= \begin{cases}2 E_{P}, & \text { for } \Delta \gg D \text { [validity range of Eq.(4)] } \\ 2 E_{P}-\frac{D^{2}}{2 E_{P}}, & \text { for } \Delta \ll D\end{cases}$

as long as $\gamma<1$. Thus, corrections due to finite bandwidth effects have to be taken into account. In particular, $E_{P}$ as obtained from Eq. (4) corresponds to the lower limit of the actual polaron binding energy.

\section{RESULTS}

\section{A. Temperature-dependent electrical resistivity and optical absorption}

Figures 2 and 3 give a brief overview on the temperature and doping dependence of the electrical and optical conductivity. The electrical resistance of the undoped $\mathrm{PrMnO}_{3}$ film is too high for a suitable measurement with our electrical setup. Therefore, only $\rho(T)$ measurements for $0.2 \leqslant x \leqslant 0.8$ are presented in Fig. 2(a).

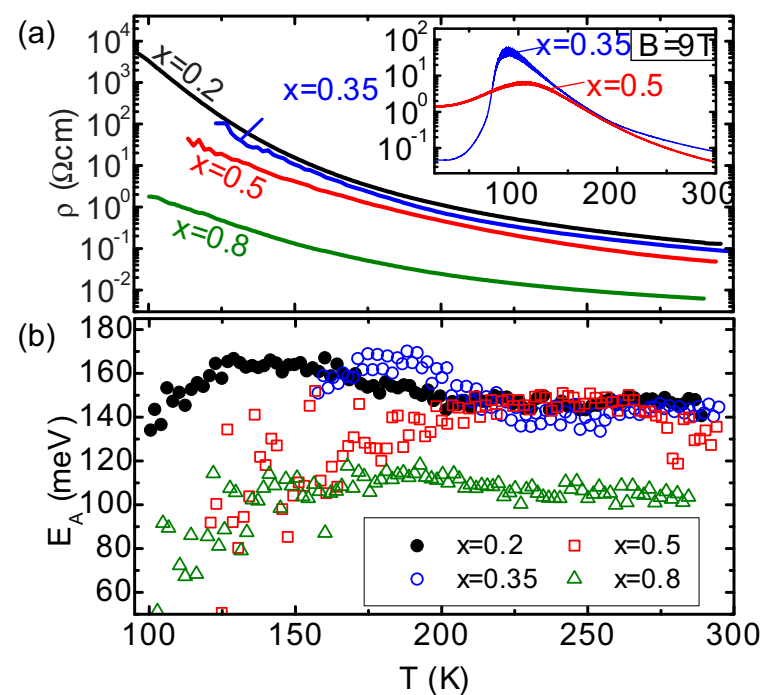

FIG. 2. (Color online) (a) Temperature-dependent resistivity of PCMO $0.2 \leqslant x \leqslant 0.8$ shows insulating behavior in the whole temperature and doping range. The sample for $x=0.35$ and $x=0.5$ exhibit a pronounced CMR (inset) at $B=9 \mathrm{~T}$. (b) The apparent activation energies calculated from resistivity via Eq. (2) within finite temperature intervals of $3 \mathrm{~K}$. The almost temperature-independent apparent energy at temperatures above $200 \mathrm{~K}$ gives the activation barrier in the paramagnetic charge-disordered state. 


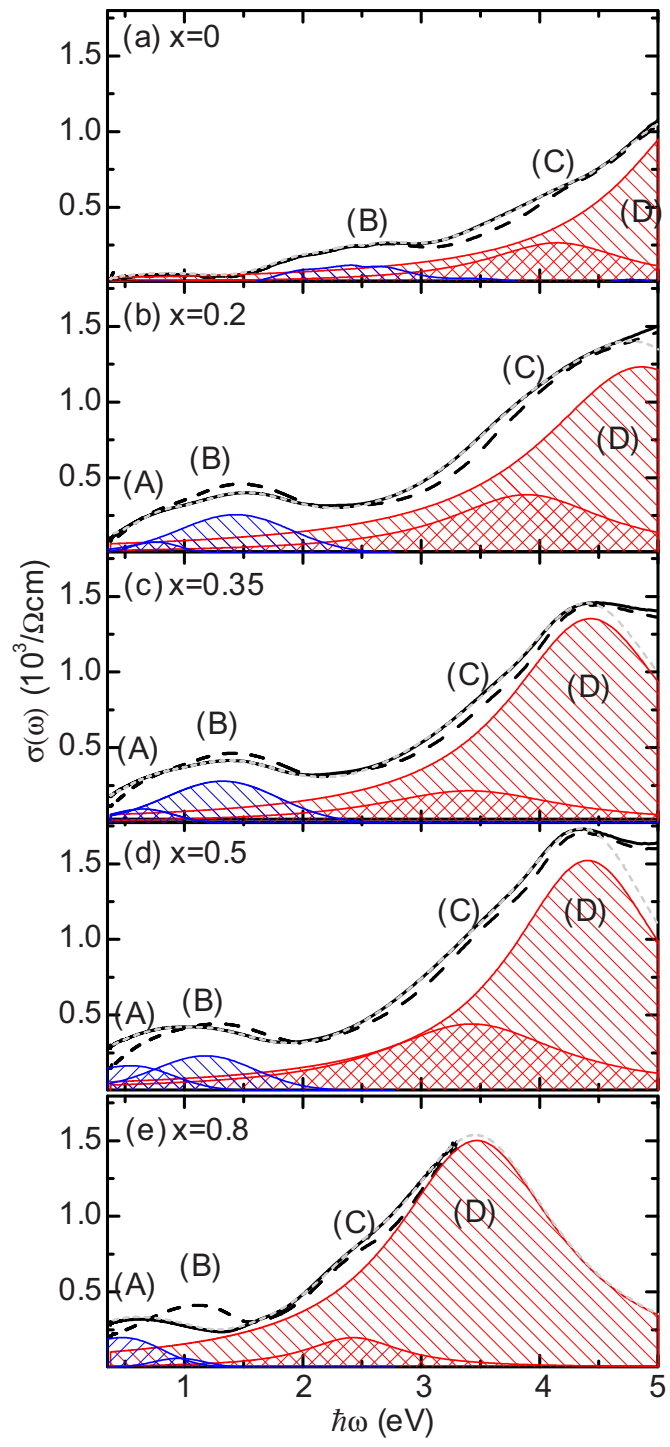

FIG. 3. (Color online) Optical conductivity of PCMO films for increasing doping level from $x=0$ (a) to $x=0.8$ (e) measured at $T=300 \mathrm{~K}$ (solid black line) and $T=80 \mathrm{~K}$ (dashed black line). The red hatched curves [peaks (C) and (D)] and blue hatched curves [peaks (A) and (B)] designate fit results from application of small polaron theory Eq. (4) to peaks (A) and (B) and Lorentzian functions to peaks (C) and (D), respectively. In the spectra (b)-(e) the sums of the fit curves are illustrated by the light-gray dashed lines.

With increasing doping level the resistivity decreases. Moreover, the $\rho(T)$ trends are consistent with thermally activated hopping polaron transport behavior [Eq. (2)]. Since the activation barrier significantly changes with the onset of charge ordering and accordingly with temperature, the apparent temperature-dependent activation energy is plotted in Fig. 2(b) [6]. The almost temperature-independent behavior of $E_{A}(T)$ for $T>200 \mathrm{~K}$ and $0.2 \leqslant x \leqslant 0.5$ is characteristic of thermal activated hopping of small polarons.

The samples with $x=0.35$ and $x=0.5$ exhibit a pronounced CMR when a magnetic field of $B=9 \mathrm{~T}$ is applied [see inset of Fig. 2(a)]. As shown in Ref. [6], the occurrence of a CMR and the presence of an ordering peak in the
$E_{A}(T)$ characteristics at $T \sim 170-200 \mathrm{~K}$ is an indication for the presence of the charge and orbital ordered (CO/OO) phase at temperatures $T<200 \mathrm{~K}$ in zero magnetic field. Note that the activation energies are not very different for the hole-doped samples but significantly lower for the nominally electron-doped sample with $x=0.8$.

Figure 3 presents the real part of the optical conductivity $\sigma(\omega)$ at room temperature and at $T=80 \mathrm{~K}$ for the PCMO films $(x=0-0.8)$. For $x=0$ we observe at least three absorption bands in the measured photon energy range: One band (labeled B) with small spectral weight centered at $\hbar \omega_{\max } \approx 2.5 \mathrm{eV}$ and two bands (C) and (D) at $\hbar \omega>4 \mathrm{eV}$, i.e., in the ultraviolet (UV) range. The feature (C) appears as a weak shoulder at the low energy tail of the intense feature (D). In the doped PCMO samples $(x \geqslant 0)$ an additional absorption band (A) appears in the NIR range. We denominate the features (A) and (B) [blue curves in Figs. 3(b)-3(e)] as NIR transitions and the features (C) and (D) (red curves in Fig. 3) as UV transitions. With increasing doping level all absorption features exhibit a pronounced shift to lower photon energies. Furthermore, the spectral weight of the UV transitions seems to increase with doping level. For $x=0.8$ the absorbance is huge for photon energies $3.5<\hbar \omega<6 \mathrm{eV}$ and out of the detector range.

Since the polaronic nature of charge carriers emerges predominantly in the low photon energy range, we focus our investigations on the NIR transitions. However, for a reliable analysis of these transitions we have to subtract the spectral weight arising from the UV transitions which partly overlap the NIR features. For this purpose we fit Lorentz oscillator functions to the bands (C) and (D) [see Fig. 3(c)]. Quantitative analysis of the fit parameters derived from Lorentz fitting of the UV features has to be handled with care because further absorption bands at higher energies, i.e., above the measured spectral range, may contribute to the spectral weight in the UV range. Thus, we restrict our analysis and discussion of the UV absorption bands to a few qualitative arguments in Appendix A of this article.

Figure 4 shows the optical conductivity $\sigma(\omega)$ at various temperatures after subtraction of the UV absorption bands. The temperature dependence of the NIR absorption is negligible for $x=0$. For $x \geqslant 0.2$ we observe a slight transfer of spectral weight to lower energies with increasing temperature. The transferred spectral weight increases with increasing doping level which for $x=0.8$ is embodied by a pronounced redshift of the NIR absorption.

\section{B. Application of the quasiclassical small polaron theory to NIR absorption bands}

As mentioned in the Introduction, we assume that the NIR features correspond to intraband and interband transitions of small polarons. Since both are expected to be phonon-assisted and the fundamental nature and shape of polaron interband and intraband transitions are very similar [44], we use the quasiclassical model for small polaron intraband absorption, i.e., Eq. (4), for the fitting of both NIR peaks.

The temperature dependence of the excitation energies, i.e., the photon energies of the maximum absorption $\hbar \omega_{\max }$ are illustrated in Fig. 5. Both peak positions exhibit similar temperature trends, i.e., a decrease with increasing temperature 


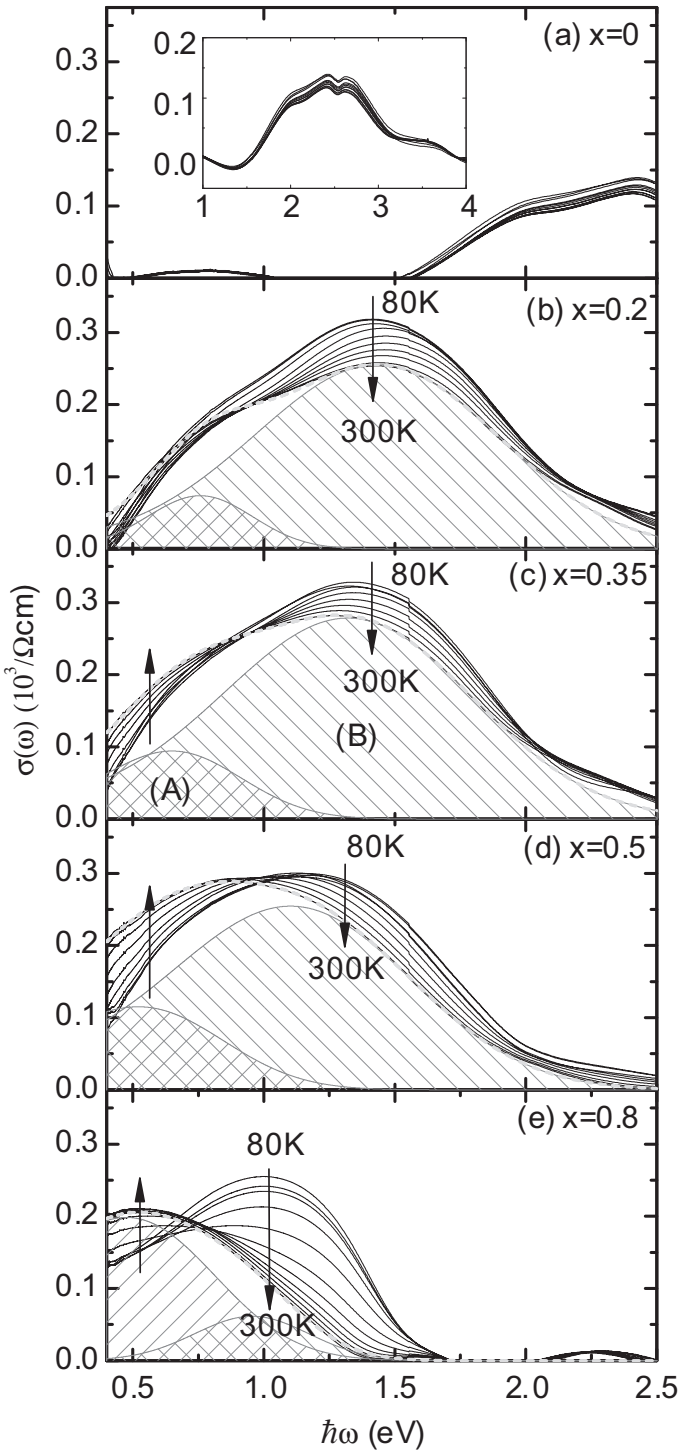

FIG. 4. Temperature dependence of the optical conductivity for increasing doping level from $x=0$ (a) to $x=0.8$ (e) after subtraction of UV absorption bands. Temperature variations from 80 to $300 \mathrm{~K}$ are indicated by arrows. The inset in (a) shows the whole spectral range of the NIR feature for $x=0$. The additional marginal absorption at $0.8 \mathrm{eV}$ may originate from defect states. This feature will not be considered in the following discussion. Hatched Gaussian shaped areas represent the fit of peaks (A) and (B) at room temperature according to Eq. (4). The light-gray dashed line is the sum of the fit curves.

and a maximum at low temperatures $(T \approx 170 \mathrm{~K})$, which seems to shift to lower temperatures with increasing hole doping. Note that for the hole-doped samples $\hbar \omega_{\max }^{(A)} \approx \frac{1}{2} \hbar \omega_{\max }^{(B)}$ at room temperature. We have, therefore, plotted $\hbar \omega_{\max }^{(A)}(T)$ and $\frac{1}{2} \hbar \omega_{\max }^{(B)}(T)$ in Fig. 5.

In the framework of the applied quasiclassical model [Eq. (4)], the peak width $\Delta$ of polaron absorption feature is related to the vibrational energy $E_{\mathrm{vib}}$ of the involved phonon mode [cf. Eq. (5)]. The temperature dependence of $E_{\text {vib }}$ determined from the fit results via Eq. (5) is depicted in Fig. 6 for both NIR absorption peaks. The vibrational

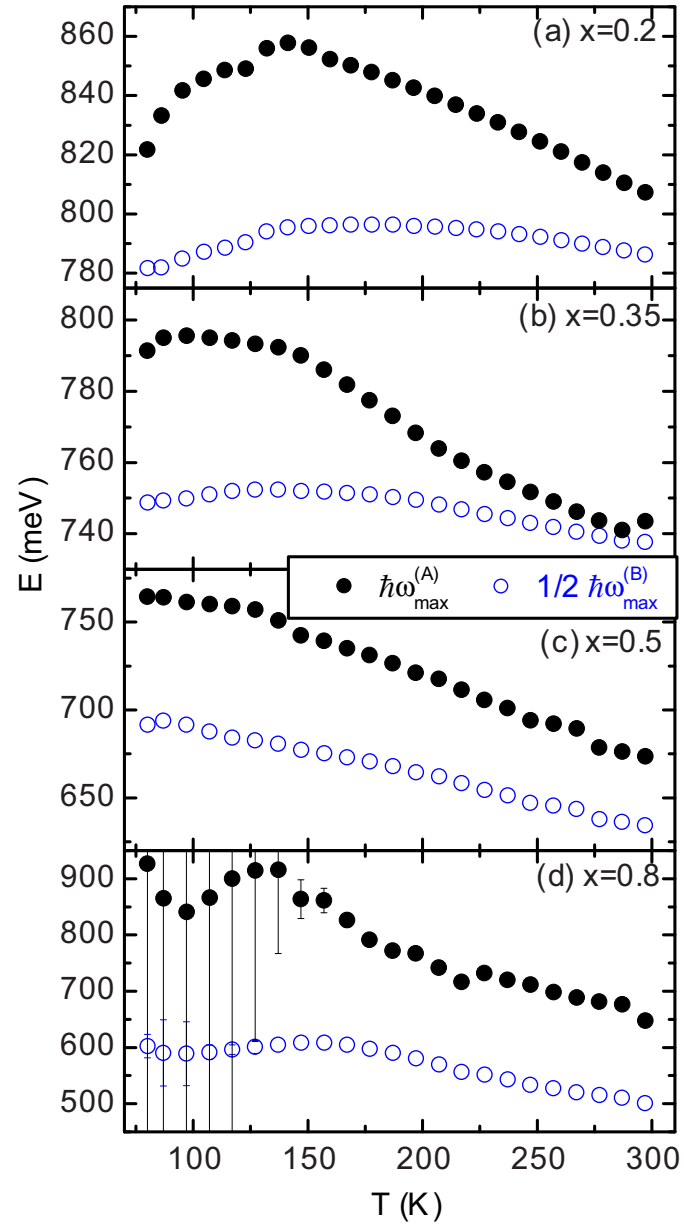

FIG. 5. (Color online) Temperature dependence of the excitation energies $\hbar \omega_{\max }^{(A)}(T)$ (solid symbols) and $\frac{1}{2} \hbar \omega_{\max }^{(B)}(T)$ (open symbols). The error bars representing the fit error are included only if they are larger than the symbol size.

energies for hole doped PCMO $(x \leqslant 0.5)$ are roughly constant at temperatures $T<\frac{\Theta_{D}}{2} \sim 150 \mathrm{~K}$, i.e., below half of the Debye temperature $\Theta_{D}$, and increase almost linearly with temperature for $T>\Theta_{D} / 2$. Some deviations from this simple behavior are observed for $x=0.2$ below $120 \mathrm{~K}$. However, the most striking difference is the entirely different temperature dependence observed for the sample with $x=0.8$.

Figure 7 summarizes the doping dependence of the fitting parameters, i.e., the excitation energy at room temperature [Fig. 7(a)], the low-temperature vibration energy at $80 \mathrm{~K}$ [Fig. 7(b)], and the spectral weights at room temperature [Fig. 7(c)]. Since the applicability of the semiclassical model on the nominally electron doped sample $(x=0.8)$ is questionable (see also Sec. V A), we have marked the fitting parameters of this sample by parentheses.

For the sample with $x=0$ we assign the absorption feature ranging from 1.5 to $4 \mathrm{eV}$ [Fig. 4(a)] to the peak (B), i.e., we expect that transitions related to peak (A) are absent (for justification, see Sec. V A). We, therefore, derive the spectral weight $(\mathrm{SW})$ via integration over the optical conductivity in the energy range $1.5<\hbar \omega<4 \mathrm{eV}$, i.e., $S W^{(B)}=\int^{\sigma}(\omega) d \omega$ and $S W^{(A)}=0$. Note that the doping dependence of the spectral weights of both NIR features is very different, $S W^{(B)}$ 


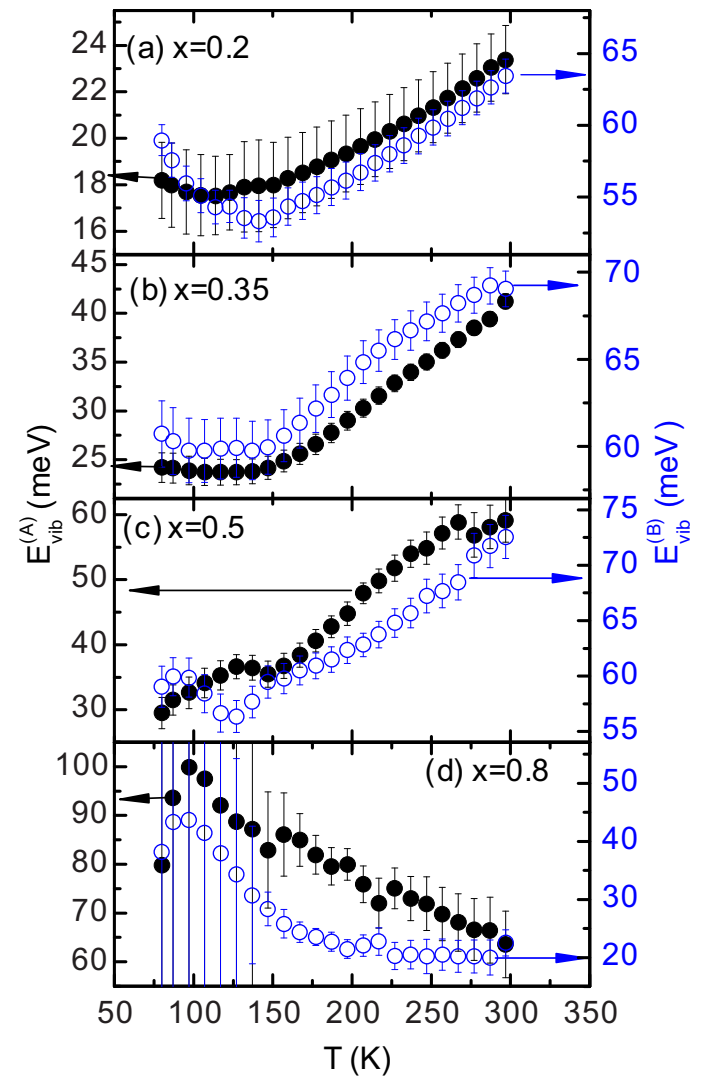

FIG. 6. (Color online) The vibrational energies $E_{\mathrm{vib}}^{(A)}(T)$ (solid symbols) and $E_{\mathrm{vib}}^{(B)}(T)$ (open symbols) as a function of temperature for $x=0.2-0.8$ calculated via Eq. (5). The significance of the fitting results is reduced for 0.8 because a substantial part of the absorption band (A) is outside the measured spectral range. Especially the fitting of the low temperature spectra of $x=0.8$ yields large error bars because peaks (A) and (B) shift together hampering their separation in the fitting procedure.

exhibits a maximum at $x=0.35$, whereas $S W^{(A)}$ increases with doping $x$.

The onset energies $E_{\text {onset }}^{(A)}$ and $E_{\text {onset }}^{(B)}$ of peaks (A) and (B) are extracted via linear extrapolation of the low energy shoulder of the fit curves according to Eq. (4). This is shown exemplarily for $x=0.35$ in Fig. 8(a). In doped PCMO samples the onset energy of peak (A) corresponds to the optical gap. The temperature dependence of the optical gap is shown in Fig. 8(b). For $x=0.2$ an optical gap $E_{\text {onset }}^{(A)}>0$ appears in the entire temperature range and exhibits only a weak temperature dependence. For $x \geqslant 0.35$ the optical gap at room temperature is small or even disappears in case of $x=0.5$ and $x=0.8$. Whereas an optical gap is absent in the whole temperature range for $x=0.8$, it reveals a strong temperature dependence in case of $x=0.35$ and $x=0.5$. For $x=0.5$ we observe a gap opening at $T \approx 200 \mathrm{~K}$. The onset energy $E_{\text {onset }}^{(B)}$ of peak (B) at $T=80 \mathrm{~K}$ as a function of doping level is shown in Fig. 8(c). For $x=0$ we obtain $E_{\text {onset }}^{(B)}$ via linear regression in the energy interval $1.6 \leqslant \hbar \omega \leqslant 1.8 \mathrm{eV}$ of the spectrum shown in Fig. 4(a). Just as the optical gap, the onset energy $E_{\text {onset }}^{(B)}$ reveals a strong doping dependence, i.e., it drastically decreases between $x=0$ and $x=0.5$ and slightly increases again for $x=0.8$.

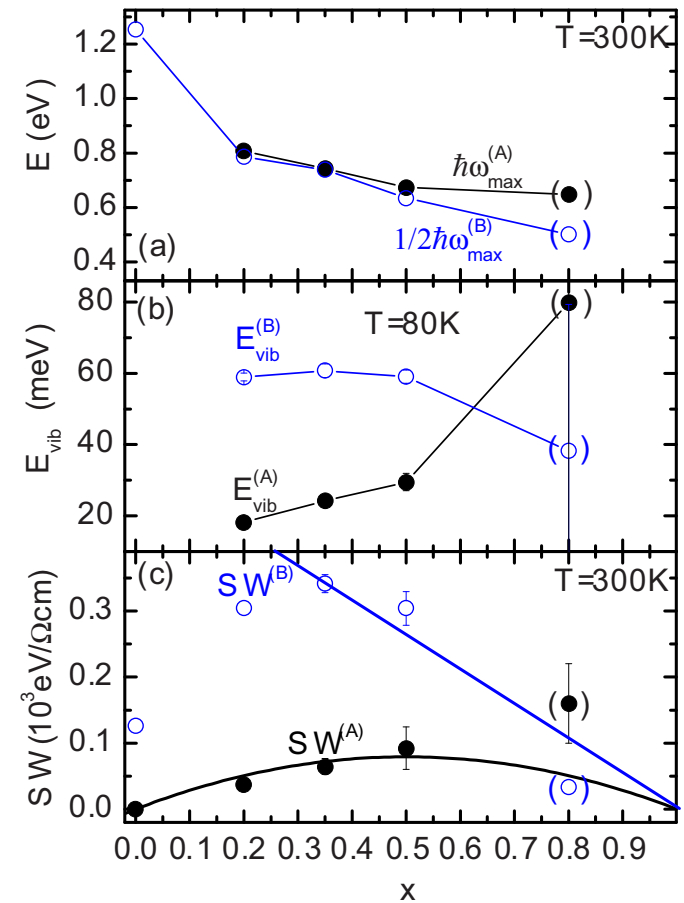

FIG. 7. (Color online) Doping dependence of (a) $\hbar \omega_{\max }^{(A)}$ and $\frac{1}{2} \hbar \omega_{\max }^{(B)}$ at room temperature and (b) $E_{\mathrm{vib}}^{(A)}$ and $E_{\mathrm{vib}}^{(B)}$ obtained via fitting feature (A) (solid symbols) and feature (B) (open symbols). The value of $\frac{1}{2} \hbar \omega_{\max }^{(B)}$ for $x=0$ is deduced from the spectral center of gravity of the NIR band and the parameters for $x=0.8$ are put in parentheses (see explanation in the text). (c) Doping dependence of the spectral weights of peak (A) (solid symbols) and (B) (open symbols) at room temperature. The black line and the blue lines in (c) represent theoretical doping dependencies according to $\sim x(1-x)$ and $\sim(1-x)$, respectively. These relations will be considered in Sec. V. The errors of the spectral weights are estimated by the spectral weight of the respective peak fit which is outside the measured spectral range, i.e., the fit area below the measurement threshold of $0.38 \mathrm{eV}$. In (a), (b), and (c) the error bars are only shown if they exceed the symbol size.

\section{DISCUSSION}

In the investigated doping and temperature range the optical absorption spectra of PCMO reveal a typical small polaron signature, i.e., peak-shaped NIR absorption bands and the absence of large polaron characteristics in terms of a Drude-like absorption feature $[14,16]$. In addition, the resistivity reveals the typical properties of small polaron mobility and, thus, the application of quasiclassical small polaron theory [Eq. (4)] in order to describe the NIR transitions seems to be reasonable. In accordance with our results, Jung et al. find two absorption bands in the NIR range of polycrystalline $\mathrm{La}_{1-x} \mathrm{Ca}_{x} \mathrm{MnO}_{3}$ single crystals which they attribute to polaron hopping between $\mathrm{Mn}^{3+}$ and $\mathrm{Mn}^{4+}$ sites and on-site JT transitions at the $\mathrm{Mn}^{3+}$ sites [26].

In order to identify the relevant transitions we have performed calculations of the electronic structure for PCMO with $x=0,0.5$, and 1 (Fig. 9). A detailed description of the electronic structure calculations will be presented in a forthcoming publication. Here we will mainly focus on the main features with respect to the absorption spectra, i.e., the 

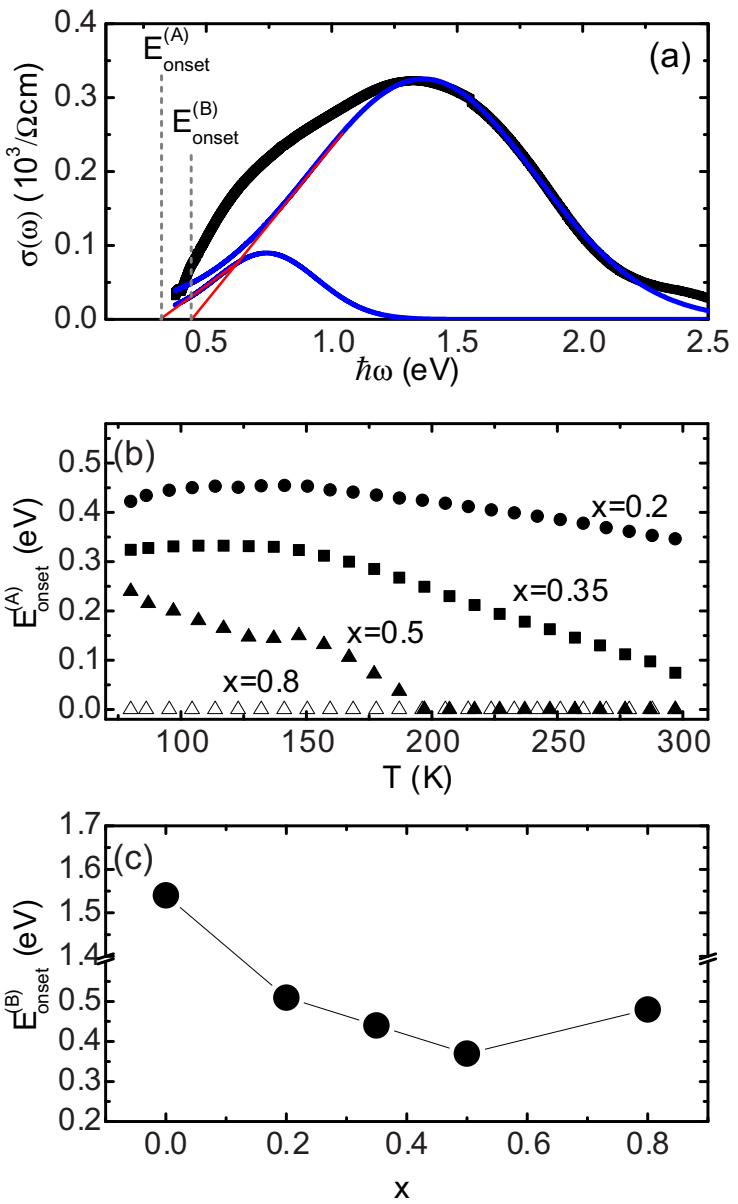

FIG. 8. (Color online) (a) Determination of the onset energies $E_{\text {onset }}^{(A)}$ and $E_{\text {onset }}^{(B)}$ via linear extrapolation of the low energy shoulder of fit curves. (b) $E_{\text {onset }}^{(A)}$ as a function of temperature for $x \geqslant 0.2$. (c) The doping dependence of $E_{\text {onset }}^{(B)}$ at $T=80 \mathrm{~K}$. Error bars are smaller than the symbol size.

band gaps, the Jahn-Teller (JT) splitting energies, and the electronic bandwidths of the valence band.

For $x=0$ and 0.5 the upper valence band edge (UVBE) is dominated by $\mathrm{O} 2 p$-Mn $e_{g}(\uparrow)$ hybrid states and the JT effect leads to a center-to-center splitting $E_{\mathrm{JT}}$ of the majority spin $\mathrm{O} 2 p-\mathrm{Mn} e_{g}(\uparrow)$ hybrid band by $E_{\mathrm{JT}} \approx 2.1 \mathrm{eV}(x=$ $0)$ and $\approx 1.7 \mathrm{eV}(x=0.5)$, respectively. The band gaps amounts to $E_{G}(x=0) \approx 1.6 \mathrm{eV}$ and $E_{G}(x=0.5)=0.6 \mathrm{eV}$. For $\mathrm{CaMnO}_{3}$ the band gap has charge transfer character with $E_{G}(x=1) \approx 1.8 \mathrm{eV}$. The main results from electronic structure calculations and a comparison with the related experimental values are summarized in Table I in Sec. V A.

According to the electronic structure calculations shown in Fig. 9 and the interpretation by Jung et al. we assign the peak (B) to on-site transitions between JT-split O $2 p-\mathrm{Mn} e_{g}(\uparrow)$ hybrid bands and the peak (A) in the NIR range to the photonassisted (intersite) hopping of small polarons. UV transitions (C) and (D) are identified as transitions between nonbonding O $2 p$ states at about $-1 \mathrm{eV}$ in Fig. 9 and minority-spin $t_{2 g}(\downarrow)$ and $e_{g}(\downarrow)$ states, respectively (details in Appendix A). These transitions are marked by the blue arrows in Fig. 9.

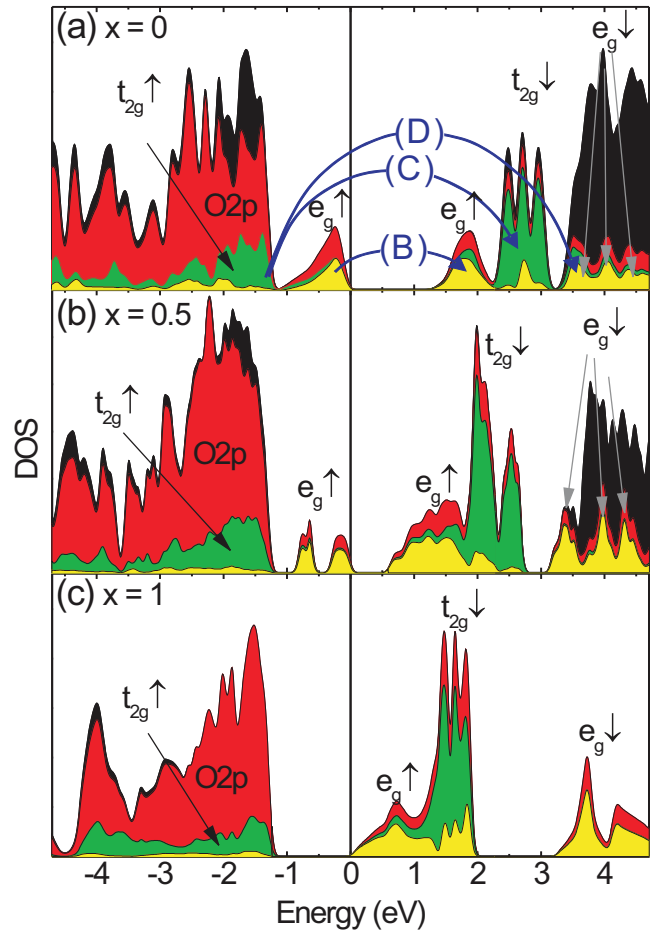

FIG. 9. (Color) Band structure PCMO for $x=0$ (top), $x=0.5$ (middle), and $x=1$ (bottom) presented as staple diagram. It reveals oxygen states (red areas) and manganese states $\left(t_{2 g}\right.$ green and $e_{g}$ yellow) near the Fermi energy. The total DOS including Pr/Ca states is illustrated in black. The blue arrows in the band structure for $x=0$ (top) indicate the optical transitions associated with peaks (B), (C), and (D) (cf. Fig. 3). We attribute the absorption peak (A) (see text) to intraband polaron hopping which cannot be illustrated in the band structure scheme.

\section{A. Quasiclassical small polaron model for PCMO}

\section{Intraband polaron hopping}

The assignment of peak (A) to photon-assisted small polaron hopping and the applicability of quasiclassical small polaron theory can be examined by comparing the optically and the thermally induced hopping. The prefactor $\sigma(0, T)$ in Eq. (4) deduced from the absorption peak (A) and the measured dc conductivities $\sigma_{\mathrm{el}}(T)=1 / \rho(T)$ [Fig. 2(a)] at room

TABLE I. Comparison of electronic and polaronic parameters: experimental (for $T=80 \mathrm{~K}$ ) and DFT results for the transfer integrals $J$ and the JT splitting energies $\hbar \omega_{\max }^{(B)}$ and $E_{\mathrm{JT}}$, the experimental onset energy of JT transitions $E_{\text {onset }}^{(B)}$, and the fundamental band gap $E_{G}$ gained from band structure calculations. The energies are given in units of $\mathrm{eV}$.

\begin{tabular}{lcccccc}
\hline \hline$x$ & $J$ (theor) & $J(\operatorname{expt})(T=300 \mathrm{~K})$ & $E_{\mathrm{JT}}$ (theor) & $\hbar \omega_{\max }^{(B)}$ & $E_{\text {onset }}^{(B)}$ & $E_{G}$ \\
\hline 0 & 0.092 & - & 2.1 & 2.4 & 1.55 & 1.6 \\
0.2 & - & 0.093 & - & 1.6 & 0.51 & - \\
0.35 & - & 0.111 & - & 1.5 & 0.44 & - \\
0.5 & 0.073 & 0.107 & 1.7 & 1.4 & 0.37 & 0.6 \\
0.8 & - & $(0.096)$ & - & 1.2 & 0.48 & - \\
1 & - & - & - & - & - & 1.8 \\
\hline \hline
\end{tabular}




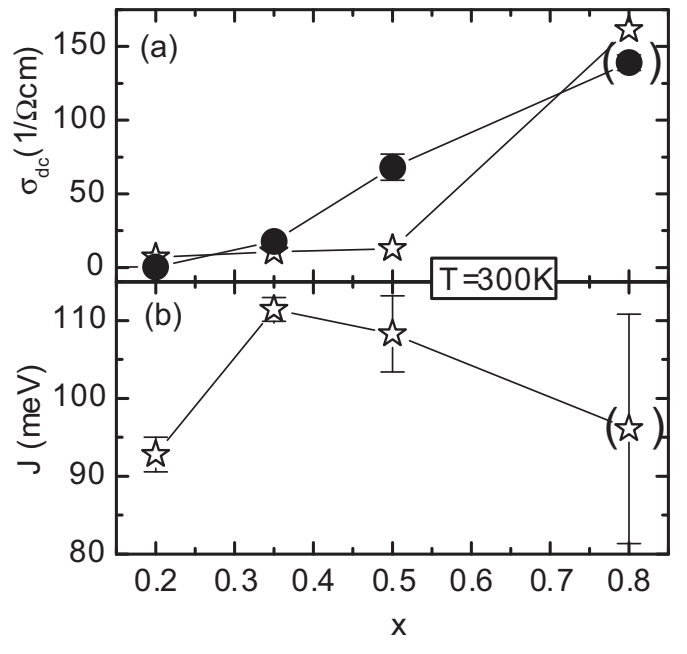

FIG. 10. Doping dependence of (a) $\sigma(0, T)$ gained from fitting the optical conductivity (black spheres) and for comparison the dc conductivity obtained via $\rho(T)$ measurement (open stars) and (b) the transfer integral $J$ determined via Eq. (7). Error bars are included if they exceed the symbol size. For $x=0.8$ the reliability of $J$ (parenthesized) is questionable.

temperature are presented in Fig. 10(a). Both conductivities agree reasonably well and reveal the same doping dependence.

Moreover, the transfer integral $J$ can be calculated from the activation barrier $E_{A}$ and the polaron binding energy $E_{P}=\frac{1}{2} \hbar \omega_{\max }^{(A)}$ according to Eq. (3) $[11,13]$. However, in doped manganites with their high charge carrier concentration the intersite Coulomb repulsion $E_{C} \approx x e^{2} /\left(12 \varepsilon_{0} \varepsilon_{r} a_{0}\right)$ have to be taken into account $[6,28]$ :

$$
J=\frac{1}{2} E_{P}-E_{A}+E_{C} .
$$

The estimated transfer integrals $J$ of the order of $90 \mathrm{meV}$ are shown in Fig. 10(b). From the electronic structure calculations we obtain bandwidth of the $\mathrm{O} 2 p$-Mn $e_{g}$ valence band of $2 D(x=0) \sim 1.1 \mathrm{eV}$ and $2 D(x=0.5) \sim 0.9 \mathrm{eV}$ which corresponds to transfer integrals $J=D / Z \sim 92$ and $73 \mathrm{meV}$, respectively (see Table I).

The consistency of the thermal and the optical conductivities as well as the agreement of experimental transfer integrals and electronic structure calculations apparently verify the applicability of the small polaron fit model and the assignment of peak (A) to polaron hopping.

In addition, the obtained vibrational energies $E_{\mathrm{vib}}^{(A)}(T)$ basically reveal the expected temperature trends, i.e., an almost temperature-independent behavior characterized by the zero-point phonon energy for $T<\frac{\Theta_{D}}{2} \sim 150 \mathrm{~K}$ and a linear increase with a slope of roughly $k T$ at higher temperatures indicating thermal phonon broadening of the polaron absorption bands. The deviation from this temperature trend for $x=0.2$, i.e., the decrease of $E_{\mathrm{vib}}^{(A)}(T)$ at low temperature may be attributed to the presence of the ferromagnetic insulating (FMI) phase at $T<180 \mathrm{~K}$. Moreover, the vibrational energies governing small polaron hopping transfer at $T=80 \mathrm{~K}$ in hole doped PCMO $(x \leqslant 0.5), E_{\mathrm{vib}}^{(A)}=\frac{1}{2} \hbar \omega_{0}^{(A)} \sim 18-30 \mathrm{meV}$, are roughly consistent with a band of vibrational Mn-O-Mn tilt modes found in the energy range of $\hbar \omega_{0} \sim 30-50 \mathrm{meV}$ in PCMO at various doping levels [18,42,43,45]. These modes change the octahedral tilting angle between nearest neighbor sites and, thus, may facilitate the intersite hopping transfer.

The spectral weight of polaron hopping transitions is assumed to follow the doping relation $\sim x(1-x)$ [26]. This theoretical doping trend is illustrated as the black line in Fig. 7(c). Polaron hopping depends on the number of occupied sites as well as unoccupied sites to which the polaron can hop. Thus, $S W^{(A)}$ should reveal a maximum at $x=0.5$, where the probability to find an occupied site next to unoccupied site is highest. For $x=0$ polaron hopping is absent [in accordance with our above made assumption that the peak (A) is absent] and the energetically lowest absorption band is associated with the interband JT transition (B). For $x \leqslant 0.5$ the spectral weight $S W^{(A)}$ follows the expected trend. We attribute the deviation for $x=0.8$ to the failure of small polaron theory for fitting the electron doped system (see details below).

(a) Limitation of the small polaron theory for fitting peak (A) due to ordering effects. The quasiclassical small polaron model [Eq. (4)] is an effective single polaron model. However, the model disregards cooperative/long-range order effects. The variety of ordered phases like CO, OO, AFM, and FM which govern the PCMO phase diagram $[46,47]$ is not taken into account. Ordering effects have probably strong impact on the hopping energy. For instance, we attribute the decrease of $\hbar \omega_{\max }^{(A)}(T)$ for $x=0.2$ with decreasing temperature below $180 \mathrm{~K}$ to the presence of a FMI phase appearing at low temperatures in low doped PCMO samples. The ferromagnetic spin coupling in the FMI phase leads to a decrease of the activation energy and accordingly the polaron binding energy [Eq. (6)] [48,49].

The increase of $\hbar \omega_{\max }^{(A)}(T)$ with decreasing temperature for $T>180 \mathrm{~K}$ is presumably due the emergence of $\mathrm{CO} / \mathrm{OO}$ (Fig. 5). CO/OO ordering hinders the intersite hopping transfer because of an additional energy barrier due to Coulomb repulsion. This barrier gives rise to a gap in the optical excitation spectrum in doping and temperature regimes with $\mathrm{CO} / \mathrm{OO}$ ordering [Fig. 8(b)]. For $x=0.2$ the persistence of the $\mathrm{OO}$ phase in the entire temperature range of $80-300 \mathrm{~K}$ leads to an optical gap of $E_{\text {onset }}^{(A)} \sim 400 \mathrm{meV}$ with marginal temperature dependence $[46,47]$. In the case of $x \geqslant 0.35$ the $\mathrm{CO} / \mathrm{OO}$-disordered phase transition manifests in the opening of an optical gap at low temperatures [50]. Since the CO/OOdisordered phase transition is of first order accompanied by a phase separation, the change of the optical gap extends over a large temperature range $[7,8]$. Despite the strong temperature induced spectral weight transfer in $x=0.8$ no optical gap develops in this sample, although a transition to a $\mathrm{CO}$ phase is expected for $T<200 \mathrm{~K}$. Note that the optical gap opening temperatures differ from the bulk charge ordering temperatures which amount to about $250 \mathrm{~K}$ for $x=0.35$ and $x=0.5$ [46,47].

As a result of gap opening, the excitation energy $\hbar \omega_{\max }^{(A)}$ for optical hopping processes increases with emerging $\mathrm{CO} / \mathrm{OO}$ ordering. This effect is not considered in the small polaron theory. Hence, the determination of the polaron binding energy $E_{P}$ from $\hbar \omega_{\max }^{(A)}$ according to Eq. (6) yields enhanced values of $E_{P}$ in doping and temperature regimes with $\mathrm{CO} / \mathrm{OO}$ ordering compared to the effective polaron binding energy. 
(b) Limitation of the small polaron theory for fitting peak (A) due to nonadiabatic and finite bandwidth effects. Furthermore, the applicability of the small polaron fit model is restricted to adiabatic hopping of polarons in the strong coupling regime when the peak width is determined by the phonon induced broadening, i.e., it requires $\gamma \leqslant 1, \lambda>1$, $D \ll \Delta$ (see Sec. III). We use the transfer integral deduced via Eq. (7) [Fig. 10(b)] for an estimate of $\gamma$ and $\lambda$. The range of $0.4 \leqslant \gamma \leqslant 0.6$ derived for $x \leqslant 0.5$ suggests that the polaron ground state is formed within the adiabatic regime. However, we find $D \approx \Delta$. Thus, both limiting cases for the determination of $E_{P}$ via Eq. (6) are not valid. The approximation $\hbar \omega_{\max }=2 E_{P}$ underestimates $E_{P}$ and thus the derived coupling parameters $\lambda$ are too small. For instance, $\lambda=\frac{E_{P}}{6 J}=0.6$ for PCMO $(x=0.35)$ is usually attributed to weak coupling according to the $\gamma-\lambda$ phase diagram provided by Fratini et al. [28]. Using the approximation $\hbar \omega_{\max }=$ $2 E_{p}-\frac{D^{2}}{2 E_{p}}$ in Eq. (4) and $D \approx 430 \mathrm{meV}$ (Fig. 9), one yields $E_{P} \approx 470 \mathrm{meV}$ and $\lambda=\frac{E_{P}}{6 J} \approx 1.1$ as an upper limit.

Furthermore, due to the underlying assumption $D \ll \Delta$, the contribution of the electronic bandwidth $2 D$ to the absorption peak width is disregarded in Eq. (4). Since $D \approx \Delta$, we overestimate the energies of the involved phonon modes and consider them only as order of magnitude estimates. With $\hbar \omega_{0}^{(A)} \sim 36-60 \mathrm{meV}$ our results are of about $20 \%$ larger than the energy of the Mn-O-Mn tilt modes found at 30-50 meV in PCMO [18,42,43,51].

Consequently, the adiabatic small polaron model Eq. (4) can be applied for yielding approximate values of the polaron binding energy and phonon modes and their relative changes as a function of temperature and doping. Polaron binding energies reported in literature are of the same order as our results, e.g., for $\mathrm{La}_{0.7} \mathrm{Ca}_{0.3} \mathrm{MnO}_{3}$ Yeh et al. have found $0.35 \mathrm{eV}$ [52]. The same polaron binding energy of $0.35 \mathrm{eV}$ is reported by Loshkareva et al. for oxygen deficient $\mathrm{CaMnO}_{3}$ [15].

For $x=0.8$, the model Eq. (4) seemingly cannot be applied to the NIR absorption features, because the optical conductivity does not show typical small polaron behavior. In particular, the spectral weight of NIR transitions is shifted to very low photon energies at room temperature. This pronounced temperature dependence resembles somehow the small to large polaron crossover characteristics such as in $\mathrm{La}_{1-x} \mathrm{Ca}_{x} \mathrm{MnO}_{3}$ and $\mathrm{La}_{1-x} \mathrm{Sr}_{x} \mathrm{MnO}_{3}[14,16]$. Since also the temperature trends of $E_{\mathrm{vib}}^{(A)}(T)$ are not consistent with a phonon assisted peak broadening which is proportional to $k T$ for $T>\frac{\Theta_{D}}{2}$, we conclude that the application of quasiclassical theory of small polaron absorption is inappropriate for PCMO with $x=0.8$.

\section{Interband on-site Jahn-Teller transitions}

As mentioned before we use Eq. (4) also for fitting peak (B) identified as on-site JT transitions, even though they are interband transitions whose excitation energy is governed by band splitting. Our results for the excitation energy $\hbar \omega_{\max }^{(B)}$ allow for an estimate of the JT energy $E_{0}$ which corresponds to the net energy gained by forming a local JT-like lattice distortion, i.e., the balance of the electronic energy gain $-2 E_{0}$ and the energy $+E_{0}$ expended for lattice deformation [53].
Disregarding the impact of on-site Coulomb interaction $U$, the electronic band width $2 D$ (strong coupling approximation for JT-polarons $D<E_{0}$ ) and ordering effects on the peak position of (B) we can get an estimate of the upper limit of $E_{0}$ from the excitation energy $\hbar \omega_{\max }^{(B)}$. The absorption maximum at $\hbar \omega_{\max }^{(B)}$ is determined by the total electronic energy difference between downshifted $\left(-2 E_{0}\right)$ and upshifted $\left(+2 E_{0}\right) \mathrm{JT}$ split states and we can determine $E_{0}$ via $E_{0}=\hbar \omega_{\max }^{(B)} / 4$. In the case of undoped $\mathrm{PrMnO}_{3}$ the JT excitation energy $\hbar \omega_{\text {max }}^{(B)}$ amounts to $\hbar \omega_{\max }^{(B)}(x=0) \sim 2.4 \mathrm{eV}$ [Fig. 7(a)]. This corresponds to a net JT energy gain of $E_{0}(x=0) \sim 0.6 \mathrm{eV}$ which is in good agreement with results obtained by other researchers for $\mathrm{LaMnO}_{3}\left(E_{0} \sim 0.5 \mathrm{eV}\right)$ [22]. The shift of peak (B) to lower photon energies with increasing $x$ [Fig. 7(a)] suggests that hole doping leads to a decrease of the JT splitting energy. For the doped PCMO samples we obtain JT energies of $E_{0}=0.2-0.4 \mathrm{eV}$, which are comparable with literature results of doped manganites [52]. For PCMO $x=0.4$ Thomas et al. report $E_{0} \approx 0.1-0.3 \mathrm{eV}$ [54]. The pronounced decrease of the excitation energy of peak (B) from $x=0$ to $x=0.2$ may be influenced by the cooperative ordering of JT distorted octahedra (OO phase) at room temperature. Note that, in general, the JT energy tends to be slightly increased in $\mathrm{CO} / \mathrm{OO}$ ordered phases (see Fig. 5).

With values of $E_{\mathrm{vib}}^{(B)}=\frac{1}{2} \hbar \omega_{0}^{(B)} \sim 50-60 \mathrm{meV}$ the vibrational energies gained from peak (B) for $0.2 \leqslant x \leqslant 0.5$ are about $50 \%$ higher than the phonon energies of symmetric JT stretching and breathing modes at $\hbar \omega_{0}=71-78 \mathrm{meV}[18,42,43,51]$. These modes affect the $\mathrm{Mn}-\mathrm{O}$ bond length and, thus, on-site transitions between JT split states. We attribute the deviation to an overestimation of the vibrational energy by the small polaron fit model [Eqs. (4) and (5)] due to the disregard of the impact of the electronic bandwidth $2 D$. Moreover, the values for the vibrational energies involved in the interband may suffer an enhanced impact of electronic bandwidth effects because the width of both, the occupied as well as the unoccupied JT band, affect the broadening of peak (B).

However, for $x=0.8$ the application of the small polaron model Eq. (4)] yields characteristic phonon energies for peak (B) of the order of $\hbar \omega_{0}=2 E_{\mathrm{vib}}^{B} \sim 200 \mathrm{meV}$ [see Fig. 6(d)] which are significantly higher than the typical phonon energies of 30-80 meV observed in manganites. Obviously Eq. (4) cannot be applied meaningfully to fit JT interband transitions in electron-doped PCMO, because JT splitting is drastically reduced in this highly Ca-doped systems or even absent. Furthermore, the strong spectral weight increase of peak (B) with decreasing temperature [Fig. 4(e)] cannot be explained in the framework of JT transitions.

In order to compare the experimental fit results with related parameters obtained from the electronic structure calculations, we summarize the JT excitation and onset energies, the electronic overlap integrals, and the band gaps in Table I. The onset energies $E_{\text {onset }}^{(B)}$ for on-site JT transitions are correlated with the electronic band gaps. For $x=0$ the experimental low temperature onset energy $E_{\text {onset }}^{(B)}(x=0) \sim 1.55 \mathrm{eV}$ nicely corresponds to the theoretical band gap $E_{G}(x=0)=$ $1.6 \mathrm{eV}$ (Fig. 9), whereas for $x=0.5$ the experimental result $E_{\text {onset }}^{(B)}(x=0.5)$ is smaller than the related theoretical band gap. However, doping trends of the band gap and the JT 
onset energy are consistent, i.e., both decrease with doping $x$ [Fig. 8(c)] due to the decrease of JT the splitting energy $E_{\mathrm{JT}}$.

The theoretical values of $E_{\mathrm{JT}}$ roughly correspond to the photon excitation energies $\hbar \omega_{\max }^{(B)}$ consumed in on-site JT transitions (peak B). From electronic structure calculations we obtain $E_{\mathrm{JT}} \approx 2.1 \mathrm{eV}(x=0)$ and $\approx 1.7 \mathrm{eV}(x=0.5)$ (between the band centers) which roughly matches the experimental values $\hbar \omega_{\max }^{(B)}(x=0) \sim 2.4 \mathrm{eV}$ and $\hbar \omega_{\max }^{(B)}(x=0.5) \sim$ $1.3 \mathrm{eV}$ [Fig. 7(a)].

In the framework of mixed valent JT-distorted $\mathrm{Mn}^{3+}$ and undistorted $\mathrm{Mn}^{4+}$ sites, the spectral weight of on-site JT transitions $S W^{(B)}$ is assumed to decrease with hole doping $x$ because the depletion of $e_{g}$ electrons reduces the number of JT split states. Accordingly, we expect a doping dependence as $S W^{(B)} \sim(1-x)$ illustrated as the blue line in Fig. 7(c) [26]. As shown in Fig. 7(c), the obtained $S W^{(B)}$ follows the expected trend for $x \geqslant 0.35$. However, for $x \leqslant 0.2$ our data deviate from the expected trend of $S W^{(B)} \sim(1-x)$ and exhibit a converse behavior. This deviation suggests a doping induced change of the transition matrix element governing the spectral weight of peak (B) which we suppose to be due to doping-dependent changes in the contribution of $\mathrm{O} 2 p$ states to the UVBE as well as changes in the octahedral tilt (see Appendix B).

\section{B. Impact of O $2 p$-Mn $3 d$ hybridization: Jahn-Teller and Zener polarons}

In the literature two alternative pictures of the nature of small polarons in hole-doped PCMO are discussed: (i) the JT polaron describing the limiting case of a site-centered carrier location involving mixed valence states $\mathrm{Mn}^{3+}$ and $\mathrm{Mn}^{4+}[16,55]$. This limit would apply if the valence band is dominated by $\mathrm{Mn} e_{g}$ states. (ii) Zener-type polarons forming dimers of almost equivalent $\mathrm{Mn}^{(3+x)+}$ sites, where the hole charge is located at the connecting $\mathrm{O}^{-}$site. Such a dimer exhibits a flattened bonding angle. Zener polarons may be relevant when the UVBE is dominated by $\mathrm{O} 2 p$ states $[3,4,56,57]$. The presence of static Zener-type polarons in the $\mathrm{CO}$ state of PCMO at $x=0.5$ was confirmed by Wu et al. via structural analysis of electron diffraction patterns [4] For $x=0.3$ see Ref. [6].

Our results reveal that both Zener and JT polaron dynamics is involved in hole doped PCMO. This is first of all suggested by the band structure in Fig. 9: The valence band in hole doped PCMO is JT split and simultaneously shows a strong contribution of $\mathrm{O} 2 p$ states. Consequently, the polaron ground state formed after switching on the electron phonon-interaction carries an electronic structure which originates from JT split Mn $e_{g}$ states as well as from the O $2 p$ orbitals. Second, the agreement of the vibrational energy $E_{\mathrm{vib}}^{(A)}$ of the phonon mode coupling to hopping transfer [peak (A)] with octahedral tilt phonon modes for $x \leqslant 0.5$ gives evidence that Zener polaron dynamics is involved in the optically induced polaron hopping [18,42,43,51]. Indeed, the obtained values for JT transitions $2 E_{\mathrm{vib}}^{(B)}$ are larger than the stretching and breathing modes at $\hbar \omega_{0}=71-78 \mathrm{meV}$. However, considering the limits of the small polaron theory [Eq. (4)] for fitting interband JT transitions (discussed in Sec. V A) the vibrational energies associated with peak (B) are consistent with the assumption that JT dynamics is involved in the on-site excitation [peak (B)] in hole doped PCMO. A further indication for the relevance of JT as well as Zener polaron dynamics, is the observation that hole doping in PCMO affects both, the number of JT split states as well as the splitting energy [Figs. 7(c) and 7(a)]. In the limiting case of mixed valence $\mathrm{Mn}^{3+}, \mathrm{Mn}^{4+}$ (JT polaron), hole doping primarily reduces the number of JT split states, i.e., $S W^{(B)} \sim(1-x)$, whereas the JT splitting energy $\hbar \omega_{\max }^{(B)}(x)$ is expected to be constant over a large doping range. In the other limit of equal $\mathrm{Mn}^{(3+x)+}$ valence states (Zener polaron), hole doping decreases the splitting energy but the amount of split states is independent of the doping level.

In the following we discuss the observed correlation $\left(\hbar \omega_{\max }^{(A)} \approx \frac{1}{2} \hbar \omega_{\max }^{(B)}\right.$ ) between the polaron hopping [peak $(\mathrm{A})$ ] and on-site JT excitation [peak (B)] [see Fig. 7(a)] in both alternative frameworks of JT and Zener polaronlike ground states. Figure 11 schematically shows the relevant initial and final states during on-site JT excitation and intersite hopping transfer.

In the picture of mixed valence ground state with pure JT polarons (Fig. 11 left panel) the correlation of the respective transition energies $\hbar \omega_{\max }^{(A)} \approx \frac{1}{2} \hbar \omega_{\max }^{(B)}$ [Fig. 7(a)] for hole doped PCMO is easily understood in the adiabatic limit. Here an electron of a JT distorted $\mathrm{Mn}^{3+}$ site is excited into an undistorted next neighbor $\mathrm{Mn}^{4+}$ unit without JT splitting. Accordingly, the optical induced hopping evolves at $\hbar \omega \approx 2 E_{P}=\frac{1}{2} \hbar \omega_{\max }^{(B)}$, i.e., the electronic contribution of the polaron binding energy corresponds to the gain in electronic energy due to JT distortion $\hbar \omega_{\max }^{(B)} / 2$. The on-site excitation evolves at $\hbar \omega \approx \hbar \omega_{\max }^{(B)}$. In the nonadiabatic regime, hopping of site-centered JT polarons requires less energy, i.e., $\hbar \omega_{\max }^{(A)}<$ $\frac{1}{2} \hbar \omega_{\max }^{(B)}$, because the JT distortion follows the transferred electron more or less immediately. This is contrary to our observation that $\hbar \omega_{\max }^{(A)} \geqslant \frac{1}{2} \hbar \omega_{\max }^{(B)}$.

In the picture of equivalent $\mathrm{Mn}^{(3+x)+}-\mathrm{Mn}^{(3+x)+}$ nearest neighbor sites with intermediate valence state, the hopping of hole states from one dimer to another involves initial and final states split by the JT effect (Fig. 11 right panel). $\hbar \omega_{\max }^{(B)}$ depends on the Mn valence state and thus continuously decreases with increasing doping level as actually revealed by Fig. 7(a). This observation strongly supports the intermediate valence scenario, depicted in the right panel of Fig. 11. Whether the transition state during hopping transfer involves a configuration, where the hole polaron is located at the $\mathrm{Mn}$ site and forms an $\mathrm{Mn}^{4+}$ intermediate, where the JT splitting is partially or totally lifted, depends on the competition between electronic and structural dynamics. In the Zener polaron picture an exact equality $\hbar \omega_{\max }^{(A)} \approx \frac{1}{2} \hbar \omega_{\max }^{(B)}$ can be most easily understood in the nonadiabatic regime, where the transition state of the hole may involve a non-JT split $\mathrm{Mn}^{4+}$ state [Fig. 11(a) right panel]. In the adiabatic limit, the slow response of the lattice on photoinduced electron hopping leads to $\hbar \omega_{\max }^{(A)}<\frac{1}{2} \hbar \omega_{\max }^{(B)}$. However, the energies of the involved octahedral tilt ( $\left.\hbar \omega_{0} \sim 30-50 \mathrm{meV}\right)$ and JT modes $\left(\hbar \omega_{0} \sim 71-78 \mathrm{meV}\right)$ are in the same order of magnitude as the electronic overlap integral $J \sim 70-100 \mathrm{meV}$ (cf. Table I in Sec. V A) and we expect that lattice deformation and electron transfer evolve at the same time scales. Consequently, neither the adiabatic nor the antiadiabatic limit is fully valid. In perspective, time-resolved optical absorption experiments 


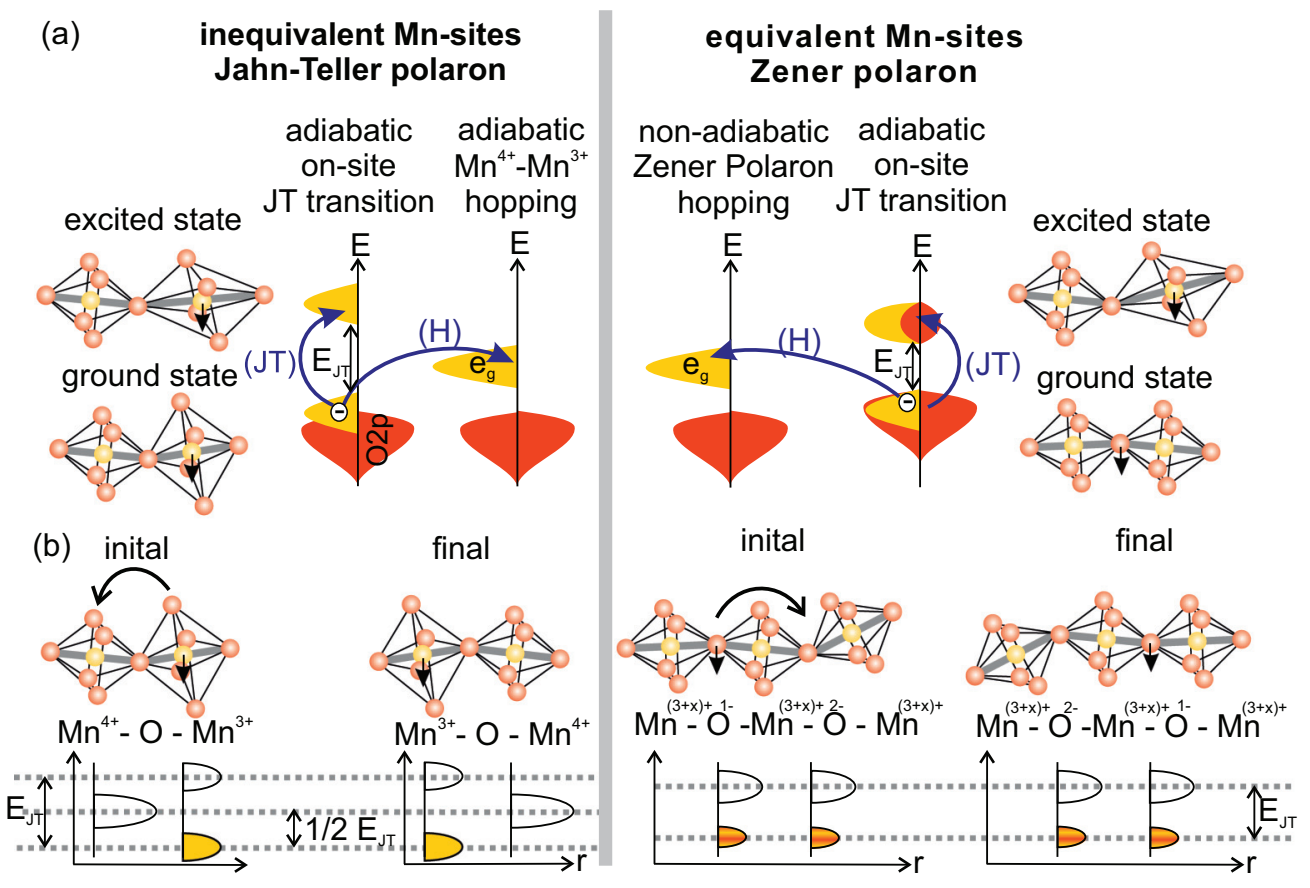

FIG. 11. (Color) Two alternative mechanisms leading to the double peak shape of $\sigma_{\mathrm{NIR}}$ and the relation $2 E_{P}=\frac{1}{2} \hbar \omega_{\max }^{(B)}$ for hole doped PCMO in the limiting pictures of mixed Mn valence states (left) and equivalent Mn valence states (right): (a) Electronic scheme of adiabatic JT on-site excitation representing a $d-d$ transition in the JT polaron picture (left). In the Zener polaron picture (right) the JT transition may have $p$ - $d$ charge transfer character. Adiabatic hopping between $\mathrm{Mn}^{3+} / \mathrm{Mn}^{4+}$ sites in the $\mathrm{JT}$ polaron picture and nonadiabatic hopping in the Zener polaron picture between equal $\mathrm{Mn}^{(3+x)+}$ sites involving a $\mathrm{Mn}^{4+}$ transition state. (b) Schematic lattice configuration and local electronic structure of the initial and final states for JT polaron hopping (left) and Zener polaron hopping (right). Notably, the JT energy in case of a Zener polaron (right) is reduced compared to the JT polaron (left).

on the femtosecond scale may give clarification on what time scale the lattice responds the electronic excitation and to what extent adiabatic and nonadiabatic effects has to be considered.

Furthermore, the energies of the JT breathing/stretching mode and the Zener-type Mn-O-Mn tilt mode are in the same order of magnitude, i.e., both dynamics couple to electronic excitations on similar time scales [58]. Thus, they cannot be independent from each other and the potential landscape of the transition state determining the hopping excitation energy $\hbar \omega_{\max }^{(\mathrm{A})}$ is assumed to be very complex. In optically excited states, the nature of the polaron, i.e., the coupling of the excited charge with phonon modes, may even change.

In addition to changes in the speed of charge and lattice dynamics, temperature-dependent deviations from the $\hbar \omega_{\max }^{(A)} \approx$ $\frac{1}{2} \hbar \omega_{\max }^{(B)}$ relation may be caused by polaron or magnetic order. For instance, $\mathrm{CO} / \mathrm{OO}$ ordering has an enhanced impact on optical intersite hopping, whereas on-site JT transition in small polaron systems are less affected by ordering effects. This leads to an increasing deviation from the observed room temperature relation $\hbar \omega_{\max }^{(A)} \approx \frac{1}{2} \hbar \omega_{\max }^{(B)}$ with decreasing temperature. For $x=0.2$, the deviation from $\hbar \omega_{\max }^{(A)} \approx \frac{1}{2} \hbar \omega_{\max }^{(B)}$ can be assigned to the $\mathrm{OO}$ phase which persists in the entire temperature range $[46,47]$.

\section{SUMMARY AND CONCLUSIONS}

In the present article we have applied quasiclassical small polaron theory describing the limiting case of the adiabatic strong coupling where the electronic level broadening is governed by phonons to fit doping and temperaturedependent NIR absorption spectra of $\operatorname{Pr}_{1-x} \mathrm{Ca}_{x} \mathrm{MnO}_{3}(x=$ $0,0.2,0.35,0.5,0.8)$ thin films. We retrieve the polaron binding energy $E_{P}$ and the characteristic phonon energy $\hbar \omega_{0}$ coupling to the polaronic state. The double peak shape of the NIR absorption band observed for all doping levels $x>0$ is interpreted as a result of optically induced intersite polaron hopping and on-site transitions between Jahn-Teller (JT) split states. The obtained JT splitting energy decreases with hole doping concentration as expected for an increasing $\mathrm{Mn}$ valence state according to $\mathrm{Mn}^{(3+x)+}$ and thus supports the presence of an intermediate Mn valence state in doped PCMO. The presence of nearly equal $\mathrm{Mn}^{(3+x)+}$ valence states in the ground state is further proven by electronic structure calculations of the electronic band structure which reveal a valence and conduction band formed by $\mathrm{O} 2 p$-Mn $e_{g}$ hybrid states which are split due to the JT effect for $x=0,0.5,1$.

The application of quasiclassical small polaron theory to both types of phonon-assisted transitions gives a number of interesting qualitative insights into the interplay of JT and Zener polaron type of dynamics as well as rough quantitative estimates of key polaron parameters. The obtained polaron binding energies are of the order of $E_{P} \sim 300-450 \mathrm{meV}$ and tend to decrease with increasing doping $\mathrm{x}$. The estimated vibrational energies coupling to the hopping transfer $\hbar \omega_{0}^{(A)} \sim$ 36-60 meV and on-site JT transitions $\hbar \omega_{0}^{(B)} \sim 100-120 \mathrm{meV}$ are significantly larger than the characteristic $\mathrm{Mn}-\mathrm{O}-\mathrm{Mn}$ tilt and $\mathrm{Mn}-\mathrm{O}$ stretching modes in PCMO, because the effect 
of a finite electronic bandwidth is disregarded in the applied analytical approximation.

Combining temperature-dependent dc-resistivity measurement which provide the determination of the activation energy $E_{A}$ with optical absorption spectra allows for an estimate of the electronic overlap integral $J$ and the electronic band width $2 D$. The obtained values $J \sim 100 \mathrm{meV}$ are roughly consistent with the theoretical calculations in Sec. V A. Absolute values for $E_{P}, E_{0}, \hbar \omega_{0}^{(A)}$, and $\hbar \omega_{0}^{(B)}$ need some corrections because $\mathrm{Pr}_{1-x} \mathrm{Ca}_{x} \mathrm{MnO}_{3}$ does not perfectly fulfill the adiabatic strong coupling limit with phonon governed peak broadening. Finite bandwidth and nonadiabatic effects in $\mathrm{Pr}_{1-x} \mathrm{Ca}_{x} \mathrm{MnO}_{3}$ result in an underestimation of $E_{P}$ and an overestimation of the characteristic phonon energies.

Ordering effects like charge, orbital, or magnetic ordering, which are not considered in the quasiclassical small polaron model, has been found to affect the apparent polaron hopping energy. Charge and orbital ordering can generate an additional barrier for next neighbor polaron hopping and can lead to the opening of an optical gap, whereas ferromagnetic ordering in $\mathrm{Pr}_{1-x} \mathrm{Ca}_{x} \mathrm{MnO}_{3}(x=0.2)$ at low temperatures facilitates hopping transport and may give rise to a decrease of the polaron binding energy $E_{P}$.

Our experimental and theoretical work advances the current research on the optical properties of polarons by giving a systematic study of the temperature and doping dependence of the polaron properties. Our results give evidence for the involvement of $\mathrm{O} 2 p-\mathrm{Mn} 3 d\left(e_{g}\right)$ states in the polaron absorption and prove the relevance of Zener as well as JahnTeller polaron dynamics in the NIR double peak absorption.

\section{ACKNOWLEDGMENTS}

The work was financially supported by the DFG via the SFB 602 project $\mathrm{A} 20$ and the SFB 1073 projects $\mathrm{B} 02$ and $\mathrm{C} 02$. We are grateful to $\mathrm{H}$. Stein for preparation of the $\mathrm{PrMnO}_{3}$ thin film and G. Busse and L. Busse for experimental support with optical absorption spectroscopy.

\section{APPENDIX A: PROPERTIES AND DOPING TRENDS OF UV TRANSITIONS}

On the basis of the presented electronic structure calculations (Fig. 9) the peaks (C) and (D) in the UV range are identified as transitions from lower $\mathrm{O} 2 p$ states $(\sim 1-2 \mathrm{eV}$ below the Fermi energy) into minority $t_{2 g}$ and $e_{g}$ states of predominant $3 d$ character. Transitions with $t_{2 g}$ final states [peak (C)] produce low spectral weight because $t_{2 g}$ orbitals are $\pi$ type and reveal a small orbital overlap with O $2 p(\sigma, \pi)$ orbitals. Thus, the transfer integral governing $\sigma / \pi \leftrightarrow \pi$ transitions is small [21]. Moreover, weak $\pi-\sigma$ type transitions into majority $e_{g}$ states may be involved at the low energy tail of the UV feature. Contrary, the spectral weight of peak (D) arising from transitions into $\sigma$-type minority $e_{g}$ states which have large orbital overlap with $\mathrm{O} 2 p(\sigma)$ orbitals is huge.
The redshift of the UV absorption bands with increasing hole concentration observed in Fig. 3 reflects the trend in the theoretical results (Fig. 9) which is probably a consequence of the decrease of Hunds coupling energy $E_{H}$, on-site exchange interaction $U$, and $\mathrm{JT}$ splitting $E_{\mathrm{JT}}$ accompanying the depletion of conduction electrons.

The temperature dependence of UV transitions is marginal in the measured temperature and doping range. Only peak (C) exhibits weak temperature dependence, i.e., its intensity increases with temperature which indicates that the optical transition $\mathrm{O} 2 p \rightarrow \mathrm{Mn} t_{2 g}$ underlying peak (C) is phonon assisted.

\section{APPENDIX B: OPTICAL MATRIX ELEMENTS FOR ON-SITE JAHN-TELLER TRANSITIONS}

We assume that the pronounced contribution of $\mathrm{O} 2 p$ states to the UVBE and hybridization with $\mathrm{Mn} e_{g}$ found in the electronic structure calculations may significantly affect the optical matrix element governing on-site JT transitions. Optical $d-d$ transitions in crystals with octahedral symmetry are generally dipole forbidden and should provoke weak intensity a factor 100-1000 smaller than intensities associated with spin and dipole allowed $\mathrm{O} 2 p$-Mn $3 d$ transfer transitions [UV transitions, peaks (C) and (D)] [15,21]. However, this is experimentally not observed in our and also other optical absorption studies which reveal rather high intensities for onsite JT transitions in manganites, i.e., even almost comparable with the intensity of UV absorption differing by at most one order of magnitude $[16,21,22,29]$. The relevance of $\mathrm{O} 2 p$ states at the UVBE in combination with a coupling to symmetry lowering vibrational Mn-O-Mn tilt modes, which is the fundament for the formation of Zener polarons, may enable dipole and spin allowed excitation channels and can lead to pronounced absorption intensities of JT transitions in agreement with experimental observations. Without coupling to phonon modes with $u$ symmetry, the on-site JT transition remains parity forbidden even if $\mathrm{O} 2 p$ states with $u$ symmetry are contributing because the JT split occupied and unoccupied electronic bands have the same symmetry. A possible scenario explaining the high intensity of JT transitions may be a different coupling of the Mn-O-Mn tilt mode to an occupied compared to an unoccupied JT split $\mathrm{O} 2 p$-Mn $e_{g}$ state. This effect can also change the degree of $\mathrm{O} 2 p$ contribution to both JT split states. In this case, the on-site JT excitation has $p$ - $d$ charge-transfer contribution which is dipole allowed.

We further assume that the optical matrix elements change with the hole concentration due to a doping-dependent lattice distortion [46] and $\mathrm{O} 2 p-\mathrm{Mn} e_{g}$ reorganization at the UVBE which cannot be understood in the framework of rigid bands [59]. As a consequence the spectral weight of on-site JT transition as a function of doping may deviate from the simple relation $\sim(1-x)$ which is just based on the electron depletion of rigid bands with increasing doping level. This is consistent with our result for $S W^{(B)}(x)$ [Fig. 7(c)].
[1] A. Lanzara, N. L. Saini, M. Brunelli, F. Natali, A. Bianconi, P. G. Radaelli, and S. W. Cheong, Phys. Rev. Lett. 81, 878 (1998).
[2] M. Jaime, M. B. Salamon, M. Rubinstein, R. E. Treece, J. S. Horwitz, and D. B. Chrisey, Phys. Rev. B 54, 11914 (1996). 
[3] Ch. Jooss, L. Wu, T. Beetz, R. F. Klie, M. Beleggia, M. A. Schofield, S. Schramm, J. Hoffmann, and Y. Zhu, Proc. Natl. Acad. Sci. USA 104, 13597 (2007).

[4] L. Wu, R. F. Klie, Y. Zhu, and Ch. Jooss, Phys. Rev. B 76, 174210 (2007).

[5] A. J. Millis, P. B. Littlewood, and B. I. Shraiman, Phys. Rev. Lett. 74, 5144 (1995).

[6] J. Hoffmann, P. Moschkau, S. Mildner, J. Norpoth, and Ch. Jooss, Mater. Res. Express 1, 046403 (2014).

[7] S. Schramm, J. Hoffmann and Ch. Jooss, J. Phys. Condens. Matter 20, 395231 (2008).

[8] G. M. Zhao, Y. S. Wang, D. J. Kang, W. Prellier, M. Rajeswari, H. Keller, T. Venkatesan, C. W. Chu, and R. L. Greene, Phys. Rev. B 62, R11949 (2000).

[9] D. Louca, T. Egami, E. L. Brosha, H. Röder, and A. R. Bishop, Phys. Rev. B 56, R8475 (1997).

[10] J. T. Devreese and A. S. Alexandrov, Rep. Prog. Phys. 72, 066501 (2009).

[11] T. Holstein, Ann. Phys. 8, 325 (1959).

[12] D. Emin, Phys. Rev. B 48, 13691 (1993).

[13] I. G. Austin and N. F. Mott, Adv. Phys. 18, 41 (1969).

[14] Ch. Hartinger, F. Mayr, A. Loidl, and T. Kopp, Phys. Rev. B 73, 024408 (2006).

[15] N. N. Loshkareva, L. V. Nomerovannaya, E. V. Mostovshchikova, A. A. Makhnev, Yu. P. Sukhorukov, N. I. Solin, T. I. Arbuzova, S. V. Naumov, N. V. Kostromitina, A. M. Balbashov, and L. N. Rybina, Phys. Rev. B 70, 224406 (2004).

[16] M. Quijada, J. Cerne, J. R. Simpson, H. D. Drew, K. H. Ahn, A. J. Millis, R. Shreekala, R. Ramesh, M. Rajeswari, and T. Venkatesan, Phys. Rev. B 58, 16093 (1998).

[17] V. N. Bogomolov and N. Mirlin, Phys. Status Solidi 27, 443 (1968).

[18] Y. Okimoto, Y. Tomioka, Y. Onose, Y. Otsuka, and Y. Tokura, Phys. Rev. B 59, 7401 (1999).

[19] G. Saucke, J. Norpoth, C. Jooss, D. Su, and Y. Zhu, Phys. Rev. B 85, 165315 (2012).

[20] N. N. Kovaleva, A. M. Oleś, A. M. Balbashov, A. Maljuk, D. N. Argyriou, G. Khaliullin, and B. Keimer, Phys. Rev. B 81, 235130 (2010).

[21] A. S. Moskvin, A. A. Makhnev, L. V. Nomerovannaya, N. N. Loshkareva, and A. M. Balbashov, Phys. Rev. B 82, 035106 (2010).

[22] J. H. Jung, K. H. Kim, D. J. Eom, T. W. Noh, E. J. Choi, J. Yu, Y. S. Kwon, and Y. Chung, Phys. Rev. B 55, 15489 (1997).

[23] J.-H. Park, C. T. Chen, S.-W. Cheong, W. Bao, G. Meigs, V. Chakarian, and Y. U. Idzerda, Phys. Rev. Lett. 76, 4215 (1996).

[24] D. Mierwaldt, S. Mildner, R. Arrigo, A. Knop-Gericke, E. Franke, A. Blumenstein, J. Hoffmann, and Ch. Jooss, Catalysts 4, 129 (2014).

[25] P. Grossmann, I. Rajkovic, R. Moré, J. Norpoth, S. Techert, C. Jooss, and K. Mann, Rev. Sci. Instrum. 83, 053110 (2012).

[26] J. H. Jung, K. H. Kim, T. W. Noh, E. J. Choi, and J. Yu, Phys. Rev. B 57, R11043 (1998).

[27] H. G. Reik and D. Heese, J. Phys. Chem. Solids 28, 581 (1967).

[28] S. Fratini and S. Ciuchi, Phys. Rev. B 74, 075101 (2006).
[29] A. Machida, Y. Moritomo, and A. Nakamura, Phys. Rev. B 58, R4281 (1998).

[30] S. Yoon, H. L. Liu, G. Schollerer, S. L. Cooper, P. D. Han, D. A. Payne, S.-W. Cheong, and Z. Fisk, Phys. Rev. B 58, 2795 (1998).

[31] P. Hohenberg and W. Kohn, Phys. Rev. 136, B864 (1964).

[32] W. Kohn and L. J. Sham, Phys. Rev. 140, A1133 (1965).

[33] P. E. Blöchl, Phys. Rev. B 50, 17953 (1994).

[34] A. D. Becke, J. Chem. Phys. 98, 1372 (1993).

[35] P. Novak, J. Kunes, L. Chaput, and W. E. Pickett, Phys. Status Solidi B 243, 563 (2006).

[36] F. Tran, P. Blaha, K. Schwarz, and P. Novak, Phys. Rev. B 74, 155108 (2006).

[37] J. P. Perdew, K. Burke, and M. Ernzerhof, Phys. Rev. Lett. 77, 3865 (1996).

[38] D. Feinberg, S. Ciuchi, and F. de Pascquale, Int. J. Mod. Phys. B 4, 1317 (1990).

[39] V. N. Bogomolov, E. K. Kudinov, and Yu. A. Firsov, Sov. Phys. Solid State 9, 2502 (1968).

[40] Y. A. Firsov and E. K. Kudinov, Phys. Solid State 43, 447 (2001).

[41] D. Emin, Adv. Phys. 24, 305 (1975).

[42] T. Kawasaki, Y. Ogimoto, N. Ogawa, K. Miyano, H. Tamaru et al., J. Appl. Phys. 101, 123714 (2007).

[43] M. Rini, R. Tobey, N. Dean, J. Itatani, Y. Tomioka, Y. Tokura, R. W. Schoenlein, and A. Cavalleri, Nature (London) 449, 72 (2007).

[44] Y. A. Firsov, Sov. Phys. Solid State 10, 1537 (1969); Semiconductors 29, 515 (1995); E. K. Kudinov and Y. A. Firsov, J. Expt. Theoret. Phys. (U.S.S.R.) 47, 601 (1965) [Sov. Phys. JETP-USSR 20, 400 (1965)].

[45] R. Sopracase, G. Gruener, E. Olive, and J.-C. Soret, Physica B: Condens. Matter 405, 45 (2010).

[46] Z. Jirak, S. Krupicka, Z. Simsa, M. Dlouha, and S. Vratislav, J. Magn. Magn. Mater. 53, 153 (1985).

[47] J. P. Hill, C. S. Nelson, M. v. Zimmermann, Y.-J. Kim, D. Gibbs, D. Casa, B. Keimer, Y. Murakami, C. Venkataraman, T. Gog, Y. Tomioka, Y. Tokura, V. Kiryukhin, T. Y. Koo, and S.-W. Cheong, Appl. Phys. A Mater. Sci. Process. 73, 723 (2001).

[48] C. Cui and T. A. Tyson, Phys. Rev. B 70, 094409 (2004).

[49] V. Markovich, I. Fita, A. I. Shames, R. Puzniak, E. Rozenberg, C. Martin, A. Wisniewski, Y. Yuzhelevskii, A. Wahl, and G. Gorodetsky, Phys. Rev. B 68, 094428 (2003).

[50] Since negative values of the optical gap obtained via linear extrapolation of the low energy shoulder of the hopping transition are physically impossible, these values were set to zero.

[51] A. Antonakos, D. Palles, E. Liarokapis, M. Filippi, and W. Prellier, J. Appl. Phys. 104, 063508 (2008).

[52] N.-C. Yeh, R. P. Vasquez, D. A. Beam, C.-C. Fu, J. Huynh and G. Beach, J. Phys. Condens. Matter 9, 3713 (1997).

[53] E. Dagotto, Nanoscale Phase Separation and Colossal Magneto-resistance: The Physics of Manganites and Related Compounds (Springer, Berlin, 2003), Vol. 136.

[54] K. J. Thomas, J. P. Hill, S. Grenier, Y-J. Kim, P. Abbamonte, L. Venema, A. Rusydi, Y. Tomioka, Y. Tokura, D. F. McMorrow, G. Sawatzky, and M. van Veenendaal, Phys. Rev. Lett. 92, 237204 (2004). 
[55] S. G. Kaplan, M. Quijada, H. D. Drew, D. B. Tanner, G. C. Xiong, R. Ramesh, C. Kwon, and T. Venkatesan, Phys. Rev. Lett. 77, 2081 (1996).

[56] P. Barone, S. Picozzi, and J. van den Brink, Phys. Rev. B 83, 233103 (2011),
[57] C. H. Patterson, Phys. Rev. B 72, 085125 (2005).

[58] C. Roos, A. Köhn, J. Gauss, and G. Diezemann, J. Chem. Phys. 141, 154110 (2014).

[59] K. Ebata, M. Hashimoto, K. Tanaka, A. Fujimori, Y. Tomioka, and Y. Tokura, Phys. Rev. B 76, 174418 (2007). 